\title{
Radiative corrections to the lepton flavor mixing in dense matter
}

\section{Jing-yu Zhu}

School of Physics and Astronomy and Tsung-Dao Lee Institute, Shanghai Jiao Tong University, Shanghai 200240, China

E-mail: zhujingyu@sjtu.edu.cn

ABSTRACT: One-loop radiative corrections will lead to a small difference between the matter potentials developed by $\nu_{\mu}$ and $\nu_{\tau}$ when they travel in a medium. By including such radiative corrections, we derive the exact expressions of the corresponding effective masssquared differences and the moduli square of the lepton flavor mixing matrix elements $\left|\widetilde{U}_{\alpha i}\right|^{2}$ (for $\alpha=e, \mu, \tau$ and $i=1,2,3$ ) in matter in the standard three-flavor mixing scheme and focus on their asymptotic behaviors when the matter density is very big (i.e., the matter effect parameter $A \equiv 2 \sqrt{2} G_{\mathrm{F}} N_{e} E$ is very big). Different from the non-trivial fixed value of $\left|\widetilde{U}_{\alpha i}\right|^{2}$ in the $A \rightarrow \infty$ limit in the case without radiative corrections, we get $\left|\widetilde{U}_{\alpha i}\right|^{2}=0$ or 1 under this extreme condition. The radiative corrections can significantly affect the lepton flavor mixing in dense matter, which are numerically and analytically discussed in detail. Furthermore, we also extend the discussion to the $(3+1)$ active-sterile neutrino mixing scheme.

Keywords: Beyond Standard Model, Neutrino Physics

ARXIV EPRINT: 2002.12182 


\section{Contents}

1 Introduction 1

2 The standard three-flavor mixing scheme 3

$2.1(\mathrm{NMO}, \nu) \quad 5$

$2.2(\mathrm{IMO}, \nu) \quad 9$

$2.3(\mathrm{NMO}, \bar{\nu}) \quad 11$

$2.4(\mathrm{IMO}, \bar{\nu}) \quad 13$

3 The $(3+1)$ flavor mixing scheme $\quad 15$

$3.1\left(\mathrm{NMO}, \nu_{3+1}\right) \quad 18$

$\begin{array}{lll}3.2 & \left(\mathrm{IMO}, \nu_{3+1}\right) & 22\end{array}$

$3.3\left(\mathrm{NMO}, \bar{\nu}_{3+1}\right) \quad 24$

$\begin{array}{lll}3.4 & \left(\mathrm{IMO}, \bar{\nu}_{3+1}\right) & 27\end{array}$

$\begin{array}{llr}4 & \text { Summary } & 28\end{array}$

\section{Introduction}

In 1978, Wolfenstein firstly pointed out that when neutrinos travel in matter, the coherent forward scattering of them with electrons and nucleons must be considered and the induced matter potentials will change the neutrino oscillation behaviors [1]. In 1985, Mikheev and Smirnov put forward that the effective mixing angle can be significantly amplified in matter (such as inside the sun) even if the corresponding mixing angle in vacuum is small. This is the wellknown Mikheev-Smirnov-Wolfenstein (MSW) effects, which successfully explain the flavor conversion behaviors of solar neutrinos in the sun [2]. Such matter effects have been proved very important in a number of reactor, solar, atmospheric, accelerator neutrino oscillation experiments aiming to accurately extract the intrinsic neutrino oscillation parameters in vacuum [3]. A lot of efforts have been made to make the neutrino oscillation probabilities in matter more intuitive [4-17]. The language of renormalization-group equation was also introduced to describe the effective neutrino masses and flavor mixing parameters in matter [18-21]. In this paper, we mainly focus on the neutrino flavor mixing in very dense matter, which has been discussed in refs. [22-25]. We further include the radiative corrections in this connection.

In the standard three-flavor mixing scheme, the Hamiltonian responsible for the propagation of neutrinos in matter can be expressed as

$$
\mathcal{H}_{\mathrm{m}}=\frac{1}{2 E} U\left(\begin{array}{ccc}
m_{1}^{2} & 0 & 0 \\
0 & m_{2}^{2} & 0 \\
0 & 0 & m_{3}^{2}
\end{array}\right) U^{\dagger}+\left(\begin{array}{ccc}
V_{e} & 0 & 0 \\
0 & V_{\mu} & 0 \\
0 & 0 & V_{\tau}
\end{array}\right) \equiv \frac{1}{2 E} \widetilde{U}\left(\begin{array}{ccc}
\widetilde{m}_{1}^{2} & 0 & 0 \\
0 & \widetilde{m}_{2}^{2} & 0 \\
0 & 0 & \widetilde{m}_{3}^{2}
\end{array}\right) \widetilde{U}^{\dagger},
$$


where $E$ is the neutrino beam energy, $m_{i}$ (for $i=1,2,3$ ) and $U$ stand respectively for neutrino masses and Pontecorvo-Maki-Nakagawa-Sakata (PMNS) lepton flavor mixing matrix, $\widetilde{m}_{i}$ (for $i=1,2,3$ ) and $\widetilde{U}$ denote effective neutrino masses and PMNS matrix in matter, respectively, and $V_{\alpha}(\alpha=e, \mu, \tau)$ represent the matter potentials arsing from charged- and neutral-current coherent forward scattering of $\nu_{\alpha}$ with electrons, protons and neutrons in matter. Considering the one-loop radiative corrections to $V_{\alpha}$, we have [26]

$$
\begin{aligned}
& V_{e}-V_{\mu}=\sqrt{2} G_{\mathrm{F}} N_{e}, \\
& V_{\tau}-V_{\mu}=-\frac{G_{\mathrm{F}}}{\sqrt{2}} \cdot \frac{3 \alpha}{2 \pi \sin ^{2} \theta_{W}} \cdot \frac{m_{\tau}^{2}}{m_{W}^{2}}\left[\left(N_{p}+N_{n}\right) \ln \left(\frac{m_{\tau}^{2}}{m_{W}^{2}}\right)+\left(N_{p}+\frac{2}{3} N_{n}\right)\right],
\end{aligned}
$$

where $G_{\mathrm{F}}$ is the Fermi coupling constant, $\alpha$ is the fine-structure constant; $N_{e}, N_{p}$ and $N_{n}$ denote the number density of electrons, protons and neutrons in matter, respectively; $m_{\tau}$ and $m_{W}$ are the masses of $\tau$ lepton and $W$ boson, respectively; and $\sin ^{2} \theta_{W} \equiv 1-m_{W}^{2} / m_{Z}^{2}$ with $m_{Z}$ being the $Z$ boson mass. To be more intuitive, eq. (1.1) can be rewritten as

$$
\mathcal{H}_{\mathrm{m}}=\frac{1}{2 E}\left[U\left(\begin{array}{ccc}
0 & 0 & 0 \\
0 & \Delta_{21} & 0 \\
0 & 0 & \Delta_{31}
\end{array}\right) U^{\dagger}+\left(\begin{array}{ccc}
A & 0 & 0 \\
0 & 0 & 0 \\
0 & 0 & A \epsilon
\end{array}\right)\right] \equiv \frac{1}{2 E}\left[\widetilde{U}\left(\begin{array}{ccc}
0 & 0 & 0 \\
0 & \widetilde{\Delta}_{21} & 0 \\
0 & 0 & \widetilde{\Delta}_{31}
\end{array}\right) \widetilde{U}^{\dagger}+B I\right],
$$

where $\Delta_{i j} \equiv m_{i}^{2}-m_{j}^{2}, \widetilde{\Delta}_{i j} \equiv \widetilde{m}_{i}^{2}-\widetilde{m}_{j}^{2}, A=2 \sqrt{2} G_{\mathrm{F}} N_{e} E, B=\widetilde{m}_{1}^{2}-m_{1}^{2}-2 E V_{\mu}$, and $I$ denotes a $3 \times 3$ identity matrix. According to eq. (1.2) and assuming $N_{e}=N_{p}=N_{n}$, $\epsilon \simeq 5 \times 10^{-5}$ is a small quantity but matters a lot in dense matter (i.e., $\mathrm{A}$ is very big).

On the other hand, given the implications of extra light sterile neutrinos in shortbaseline neutrino oscillation experiments [27], we extend our discussion to the scheme of $(3+1)$ flavor mixing with one more sterile neutrino $\nu_{s}$. The corresponding Hamiltonian describing the propagation of neutrinos in a medium turns out to be

$$
\begin{aligned}
\mathcal{H}_{\mathrm{m}}^{s} & =\frac{1}{2 E}\left[V\left(\begin{array}{cccc}
0 & 0 & 0 & 0 \\
0 & \Delta_{21} & 0 & 0 \\
0 & 0 & \Delta_{31} & 0 \\
0 & 0 & 0 & \Delta_{41}
\end{array}\right) V^{\dagger}+\left(\begin{array}{cccc}
A & 0 & 0 & 0 \\
0 & 0 & 0 & 0 \\
0 & 0 & A \epsilon & 0 \\
0 & 0 & 0 & A^{\prime}
\end{array}\right)\right] \\
& =\frac{1}{2 E}\left[\widetilde{V}\left(\begin{array}{cccc}
0 & 0 & 0 & 0 \\
0 & \widetilde{\Delta}_{21} & 0 & 0 \\
0 & 0 & \widetilde{\Delta}_{31} & 0 \\
0 & 0 & 0 & \widetilde{\Delta}_{41}
\end{array}\right) \widetilde{V}^{\dagger}+B I\right],
\end{aligned}
$$

where $V$ and $\widetilde{V}$ denote the $4 \times 4$ lepton flavor mixing matrix in vacuum and matter, respectively, $A^{\prime}=-2 E V_{\mu}=\sqrt{2} G_{\mathrm{F}} N_{n} E, \Delta_{41}=m_{4}^{2}-m_{1}^{2}$, and $\widetilde{\Delta}_{41}=\widetilde{m}_{4}^{2}-\widetilde{m}_{1}^{2}$ with $m_{4}$ and $\widetilde{m}_{4}$ being the sterile neutrino mass in vacuum and matter, respectively. The existence of an extra sterile neutrino can make the neutrino flavor mixing in dense matter very different from the standard three-flavor mixing scheme.

The remaining parts of this paper are organized as follows. In section 2, we include the radiative corrections and derive the corresponding expressions of $\widetilde{\Delta}_{i j},\left|\widetilde{U}_{\alpha i}\right|^{2}$ and $\widetilde{U}_{\alpha i} \widetilde{U}_{\beta i}^{*}$ in 
matter in the standard three-flavor mixing scheme. The asymptotic behaviors of $\widetilde{\Delta}_{i j}$ and $\left|\widetilde{U}_{\alpha i}\right|^{2}$ and neutrino oscillations in dense matter are analytically and numerically investigated in detail. In section 3 , we extend our discussion to the $(3+1)$ flavor mixing scheme. Section 4 is devoted to a brief summary.

\section{The standard three-flavor mixing scheme}

In the standard three-flavor mixing scheme, the exact formulas of $\widetilde{\Delta}_{i j}$ without radiative corrections have been given in refs. [28-30]. Considering radiative correction effects, we derive the eigenvalues of $\mathcal{H}_{\mathrm{m}}$ in eq. (1.3) and express the two independent effective masssquared differences $\widetilde{\Delta}_{i j}$ (for $\left.i j=21,31\right)$ as

$$
\begin{aligned}
& \widetilde{\Delta}_{21}=\frac{2}{3} \sqrt{x^{2}-3 y} \sqrt{3\left(1-z^{2}\right)}, \\
& \widetilde{\Delta}_{31}=\frac{1}{3} \sqrt{x^{2}-3 y}\left[3 z+\sqrt{3\left(1-z^{2}\right)}\right],
\end{aligned}
$$

for the case of normal mass ordering (NMO) with $m_{1}<m_{2}<m_{3}$; or

$$
\begin{aligned}
& \widetilde{\Delta}_{21}=\frac{1}{3} \sqrt{x^{2}-3 y}\left[3 z-\sqrt{3\left(1-z^{2}\right)}\right], \\
& \widetilde{\Delta}_{31}=-\frac{2}{3} \sqrt{x^{2}-3 y} \sqrt{3\left(1-z^{2}\right)}
\end{aligned}
$$

for the case of inverted mass ordering (IMO) case with $m_{3}<m_{1}<m_{2}$, where

$$
\begin{aligned}
x= & \Delta_{21}+\Delta_{31}+A(1+\epsilon), \\
y= & \Delta_{21} \Delta_{31}+A\left\{\Delta_{21}\left[1-\left|U_{e 2}\right|^{2}+\epsilon\left(1-\left|U_{\tau 2}\right|^{2}\right)\right]\right. \\
& \left.+\Delta_{31}\left[\left(1-\left|U_{e 3}\right|^{2}\right)+\epsilon\left(1-\left|U_{\tau 3}\right|^{2}\right)\right]\right\}+A^{2} \epsilon, \\
z= & \cos \left[\frac{1}{3} \arccos \frac{2 x^{3}-9 x y+27 d}{2 \sqrt{\left(x^{2}-3 y\right)^{3}}}\right]
\end{aligned}
$$

with $d=A \Delta_{21} \Delta_{31}\left(\left|U_{e 1}\right|^{2}+\epsilon\left|U_{\tau 1}\right|^{2}\right)+A^{2} \epsilon\left(\left|U_{\mu 2}\right|^{2} \Delta_{21}+\left|U_{\mu 3}\right|^{2} \Delta_{31}\right)$. Taking the trace of $\mathcal{H}_{\mathrm{m}}$ yields

$$
B=\frac{1}{3}\left[\Delta_{21}+\Delta_{31}+A(1+\epsilon)-\widetilde{\Delta}_{21}-\widetilde{\Delta}_{31}\right] .
$$

The unitarity conditions of $\widetilde{U}$ and the sum rules derived from $\mathcal{H}_{\mathrm{m}}$ and $\mathcal{H}_{\mathrm{m}}^{2}$, constitute a set of linear equations of $\widetilde{U}_{\alpha i} \widetilde{U}_{\beta i}^{*}$ (for $\alpha, \beta=e, \mu, \tau$ and $i=1,2,3$ ):

$$
\begin{aligned}
\sum_{i} \widetilde{U}_{\alpha i} \widetilde{U}_{\beta i}^{*} & =\delta_{\alpha \beta} \\
\sum_{i} \widetilde{\Delta}_{i 1} \widetilde{U}_{\alpha i} \widetilde{U}_{\beta i}^{*} & =\sum_{i} \Delta_{i 1} U_{\alpha i} U_{\beta i}^{*}+\mathcal{A}_{\alpha \beta}-B \delta_{\alpha \beta} \\
\sum_{i} \widetilde{\Delta}_{i 1}\left(\widetilde{\Delta}_{i 1}+2 B\right) \widetilde{U}_{\alpha i} \widetilde{U}_{\beta i}^{*} & =\sum_{i} \Delta_{i 1}\left(\Delta_{i 1}+\mathcal{A}_{\alpha \alpha}+\mathcal{A}_{\beta \beta}\right) U_{\alpha i} U_{\beta i}^{*}+\mathcal{A}_{\alpha \beta}^{2}-B^{2} \delta_{\alpha \beta}
\end{aligned}
$$


where $\mathcal{A}_{\alpha \beta}$ stand for the $(\alpha, \beta)$ element of the matter potential matrix $\mathcal{A} \equiv \operatorname{Diag}\{A, 0, A \epsilon\}$. Taking $\alpha=\beta$ and solving eq. (2.5), we obtain

$$
\left|\widetilde{U}_{\alpha i}\right|^{2}=\frac{1}{\prod_{k \neq i} \widetilde{\Delta}_{i k}} \sum_{j}\left(F_{\alpha}^{i j}\left|U_{\alpha j}\right|^{2}\right)
$$

with

$$
F_{\alpha}^{i j}=\prod_{k \neq i}\left(\Delta_{j 1}-\widetilde{\Delta}_{k 1}-B+\mathcal{A}_{\alpha \alpha}\right)
$$

where $\alpha=e, \mu, \tau$ and $i, j, k=1,2,3$. Similarly, in the case of $\alpha \neq \beta, \widetilde{U}_{\alpha i} \widetilde{U}_{\beta i}^{*}$ can be derived from eq. (2.5):

$$
\widetilde{U}_{\alpha i} \widetilde{U}_{\beta i}^{*}=\frac{1}{\prod_{k \neq i} \widetilde{\Delta}_{i k}} \sum_{m=1,2}\left(F_{\alpha \beta}^{i m} U_{\alpha m} U_{\beta m}^{*}\right)
$$

with

$$
F_{\alpha \beta}^{i m}=\Delta_{3 m}\left(\Delta_{n 1}-\widetilde{\Delta}_{i 1}-B+\mathcal{A}_{\gamma \gamma}\right)
$$

where $(\alpha, \beta, \gamma)$ run over $(e, \mu, \tau)$ and $n \neq m=1,2$. Note that $\widetilde{U}_{\alpha 1} \widetilde{U}_{\beta 1}^{*}, \widetilde{U}_{\alpha 2} \widetilde{U}_{\beta 2}^{*}$ and $\widetilde{U}_{\alpha 3} \widetilde{U}_{\beta 3}^{*}$ for $\alpha \neq \beta$ constitute the effective Dirac leptonic unitarity triangle in the complex plane. From eq. (2.8), it is straightforward to check that the Naumov relation $\widetilde{\mathcal{J}} \widetilde{\Delta}_{21} \widetilde{\Delta}_{31} \widetilde{\Delta}_{32}=$ $\mathcal{J} \Delta_{21} \Delta_{31} \Delta_{32}$ [31] still holds, where $\mathcal{J}$ and $\widetilde{\mathcal{J}}$ are the Jarlskog invariants [32] in vacuum and in matter, respectively,

$$
\begin{aligned}
\operatorname{Im}\left(U_{\alpha i} U_{\beta j} U_{\alpha j}^{*} U_{\beta i}^{*}\right) & =\mathcal{J} \sum_{\gamma} \varepsilon_{\alpha \beta \gamma} \sum_{k} \varepsilon_{i j k}, \\
\operatorname{Im}\left(\widetilde{U}_{\alpha i} \widetilde{U}_{\beta j} \widetilde{U}_{\alpha j}^{*} \widetilde{U}_{\beta i}^{*}\right) & =\widetilde{\mathcal{J}} \sum_{\gamma} \varepsilon_{\alpha \beta \gamma} \sum_{k} \varepsilon_{i j k},
\end{aligned}
$$

with $\varepsilon_{\alpha \beta \gamma}$ and $\varepsilon_{i j k}$ being three-dimension Levi-Civita symbols. The only difference due to the radiative corrections in the exact formulas of $\widetilde{\Delta}_{i j},\left|\widetilde{U}_{\alpha i}\right|^{2}$ and $\widetilde{U}_{\alpha i} \widetilde{U}_{\beta i}^{*}$ above is the appearance of the term $\mathcal{A}_{\tau \tau}=A \epsilon$. By setting $\epsilon=0$, one can turn off the radiative corrections and get the corresponding expressions of $\widetilde{\Delta}_{i j},\left|\widetilde{U}_{\alpha i}\right|^{2}$ and $\widetilde{U}_{\alpha i} \widetilde{U}_{\beta i}^{*}$ in the previous literature [30,33-35]. With the help of eqs. (2.1), (2.2) and (2.8), we can directly write out the probabilities of the $\nu_{\alpha} \rightarrow \nu_{\beta}$ (for $\alpha, \beta=e, \mu, \tau$ ) oscillations in matter

$$
\begin{aligned}
\widetilde{P}_{\alpha \beta}= & \delta_{\alpha \beta}-4 \sum_{i<j} \operatorname{Re}\left(\widetilde{U}_{\alpha i} \widetilde{U}_{\beta i}^{*} \widetilde{U}_{\alpha j}^{*} \widetilde{U}_{\beta j}\right) \sin ^{2}\left(\frac{\widetilde{\Delta}_{j i} L}{4 E}\right) \\
& +2 \sum_{i<j} \operatorname{Im}\left(\widetilde{U}_{\alpha i} \widetilde{U}_{\beta i}^{*} \widetilde{U}_{\alpha j}^{*} \widetilde{U}_{\beta j}\right) \sin \left(\frac{\widetilde{\Delta}_{j i} L}{2 E}\right),
\end{aligned}
$$

where $\alpha, \beta=e, \mu, \tau ; i, j=1,2,3$; and $L$ is the neutrino oscillation length. Note that the results in eqs. (2.1)-(2.11) are only valid for a neutrino beam. When it comes to an antineutrino beam, we need to do the replacements $U \rightarrow U^{*}$ and $A \rightarrow-A$. According to the exact expressions of $\widetilde{\Delta}_{i j},\left|\widetilde{U}_{\alpha i}\right|^{2}$ and $\widetilde{U}_{\alpha i} \widetilde{U}_{\beta i}^{*}$ in eqs. (2.1), (2.2) (2.6) and (2.8), we study 
the neutrino flavor mixing in dense matter in the standard three-flavor mixing scheme. Both neutrinos and antineutrinos with the normal or inverted mass ordering (i.e., cases $(\mathrm{NMO}, \nu),(\mathrm{IMO}, \nu),(\mathrm{NMO}, \bar{\nu})$ and $(\mathrm{IMO}, \bar{\nu}))$ will be considered separately. Numerically, we take the standard parametrization of $U$,

$$
U=\left(\begin{array}{ccc}
c_{12} c_{13} & s_{12} c_{13} & s_{13} e^{-\mathrm{i} \delta} \\
-s_{12} c_{23}-c_{12} s_{13} s_{23} e^{\mathrm{i} \delta} & c_{12} c_{23}-s_{12} s_{13} s_{23} e^{\mathrm{i} \delta} & c_{13} s_{23} \\
s_{12} s_{23}-c_{12} s_{13} c_{23} e^{\mathrm{i} \delta} & -c_{12} s_{23}-s_{12} s_{13} c_{23} e^{\mathrm{i} \delta} & c_{13} c_{23}
\end{array}\right),
$$

and input the best-fit values of $\left(\theta_{12}, \theta_{13}, \theta_{23}, \delta, \Delta_{21}, \Delta_{31}\right)$ in refs. [36, 37]:

- NMO: $\theta_{12}=33.82^{\circ}, \theta_{13}=8.60^{\circ}, \theta_{23}=48.6^{\circ}, \delta=221^{\circ}, \Delta_{21}=7.39 \times 10^{-5} \mathrm{eV}^{2}$ and $\Delta_{31}=2.528 \times 10^{-3} \mathrm{eV}^{2}$

- IMO: $\theta_{12}=33.82^{\circ}, \theta_{13}=8.64^{\circ}, \theta_{23}=48.8^{\circ}, \delta=282^{\circ}, \Delta_{21}=7.39 \times 10^{-5} \mathrm{eV}^{2}$ and $\Delta_{31}=-2.436 \times 10^{-3} \mathrm{eV}^{2}$.

Analytically, we treat $\Delta_{21} / A, \Delta_{31} / A$ and $\epsilon$ as small quantities and make perturbative expansions of $\widetilde{\Delta}_{i j}$ and $\left|\widetilde{U}_{\alpha i}\right|^{2}$. Thus the analytical approximations in this section only apply to the range $A \gg \Delta_{31}$.

\section{$2.1(\mathrm{NMO}, \nu)$}

Let us first consider the case of a neutrino beam with normal mass ordering. The corresponding evolution of $\widetilde{\Delta}_{i j}$ and $\left|\widetilde{U}_{\alpha i}\right|^{2}$ with the matter effect parameter $A$ are illustrated in the upper left panel of figure 1 and figure 2, respectively. We find that the radiative corrections may significantly affect the values of $\widetilde{\Delta}_{i j}$ and $\left|\widetilde{U}_{\alpha i}\right|^{2}$ only if $A$ is big enough (for example, $A>1 \mathrm{eV}^{2}$ ). This can be revealed more clearly by expanding the exact expressions of $\widetilde{\Delta}_{i j}$ and $\left|\widetilde{U}_{\alpha i}\right|^{2}$ in terms of $\Delta_{21} / A, \Delta_{31} / A$ and $\epsilon$. Only keeping the first order of these quantities, we simplify $\widetilde{\Delta}_{i j}$ in eq. (2.1) as

$$
\begin{aligned}
& \widetilde{\Delta}_{21} \simeq \xi, \\
& \widetilde{\Delta}_{31} \simeq A-\frac{1}{2}\left[A \epsilon+\left(1-3\left|U_{e 2}\right|^{2}\right) \Delta_{21}+\left(1-3\left|U_{e 3}\right|^{2}\right) \Delta_{31}-\xi\right],
\end{aligned}
$$

with

$$
\begin{aligned}
\xi= & \left\{\left[\left(1-\left|U_{e 2}\right|^{2}\right) \Delta_{21}+\left(1-\left|U_{e 3}\right|^{2}\right) \Delta_{31}+A \epsilon\right]^{2}-4 A \epsilon\left(\left|U_{\mu 2}\right|^{2} \Delta_{21}+\left|U_{\mu 3}\right|^{2} \Delta_{31}\right)\right. \\
& \left.-4\left|U_{e 1}\right|^{2} \Delta_{21} \Delta_{31}\right\}^{1 / 2} .
\end{aligned}
$$

According to eq. (2.13), it becomes clear that if $A$ is big enough, $\widetilde{\Delta}_{21}$ will increase with $A$ instead of taking a fixed value in the case without radiative corrections. The reason why the radiation corrections to $\widetilde{\Delta}_{31}$ are not significant is just that the much smaller $A \epsilon$ term appears in the next-to-leading order with the leading order being $A$. By performing 

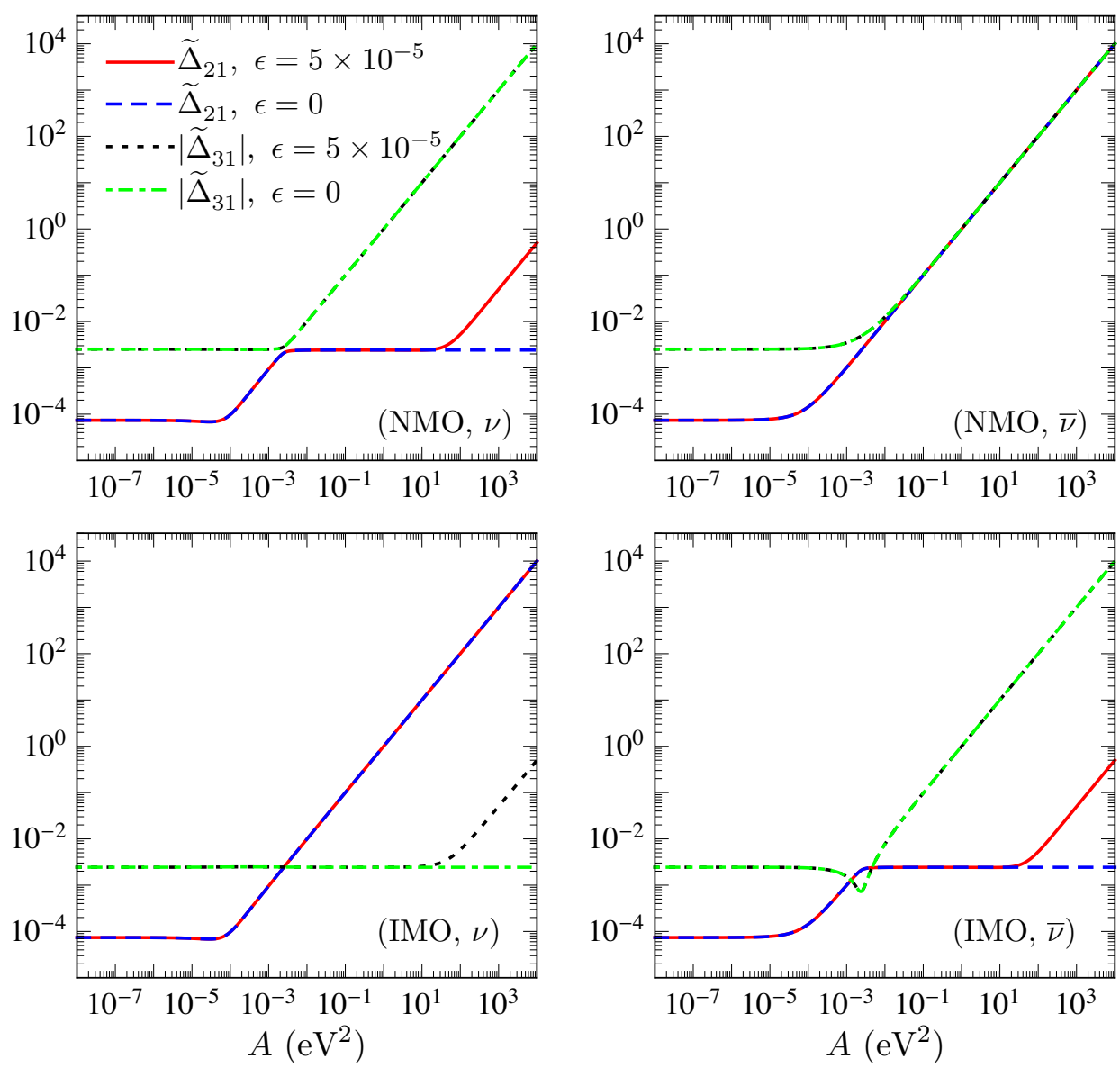

Figure 1. In the standard three-flavor mixing scheme, the illustration of how the effective neutrino mass-squared differences $\widetilde{\Delta}_{21}$ and $\left|\widetilde{\Delta}_{31}\right|$ evolve with the matter effect parameter $A$ in the cases with or without radiative corrections, where the best-fit values of $\left(\theta_{12}, \theta_{13}, \theta_{23}, \delta, \Delta_{21}, \Delta_{31}\right)$ in refs. [36, 37] have been input.

perturbative expansions of eq. (2.6) in terms of $\Delta_{21} / A, \Delta_{31} / A$ and $\epsilon$ and only keeping the leading order, $\left|\widetilde{U}_{\alpha i}\right|^{2}$ are approximately expressed as

$$
\begin{aligned}
& \left|\widetilde{U}_{e 1}\right|^{2} \simeq\left|\widetilde{U}_{e 2}\right|^{2} \simeq\left|\widetilde{U}_{\mu 3}\right|^{2} \simeq\left|\widetilde{U}_{\tau 3}\right|^{2} \simeq 0, \quad\left|\widetilde{U}_{e 3}\right|^{2} \simeq 1 \\
& \left|\widetilde{U}_{\mu 1}\right|^{2} \simeq\left|\widetilde{U}_{\tau 2}\right|^{2} \simeq \frac{1}{2}+\frac{A \epsilon+\Delta_{21}\left(\left|U_{\tau 2}\right|^{2}-\left|U_{\mu 2}\right|^{2}\right)+\Delta_{31}\left(\left|U_{\tau 3}\right|^{2}-\left|U_{\mu 3}\right|^{2}\right)}{2 \xi} \\
& \left|\widetilde{U}_{\mu 2}\right|^{2} \simeq\left|\widetilde{U}_{\tau 1}\right|^{2} \simeq \frac{1}{2}-\frac{A \epsilon+\Delta_{21}\left(\left|U_{\tau 2}\right|^{2}-\left|U_{\mu 2}\right|^{2}\right)+\Delta_{31}\left(\left|U_{\tau 3}\right|^{2}-\left|U_{\mu 3}\right|^{2}\right)}{2 \xi}
\end{aligned}
$$

This means that the neutrino flavor mixing in dense matter can be approximately described by only one degree of freedom, as having been pointed out in ref. [24]:

$$
\left.\widetilde{U}\right|_{A \gg \Delta_{31}} \simeq\left(\begin{array}{ccc}
0 & 0 & 1 \\
\cos \theta & \sin \theta & 0 \\
-\sin \theta & \cos \theta & 0
\end{array}\right),
$$



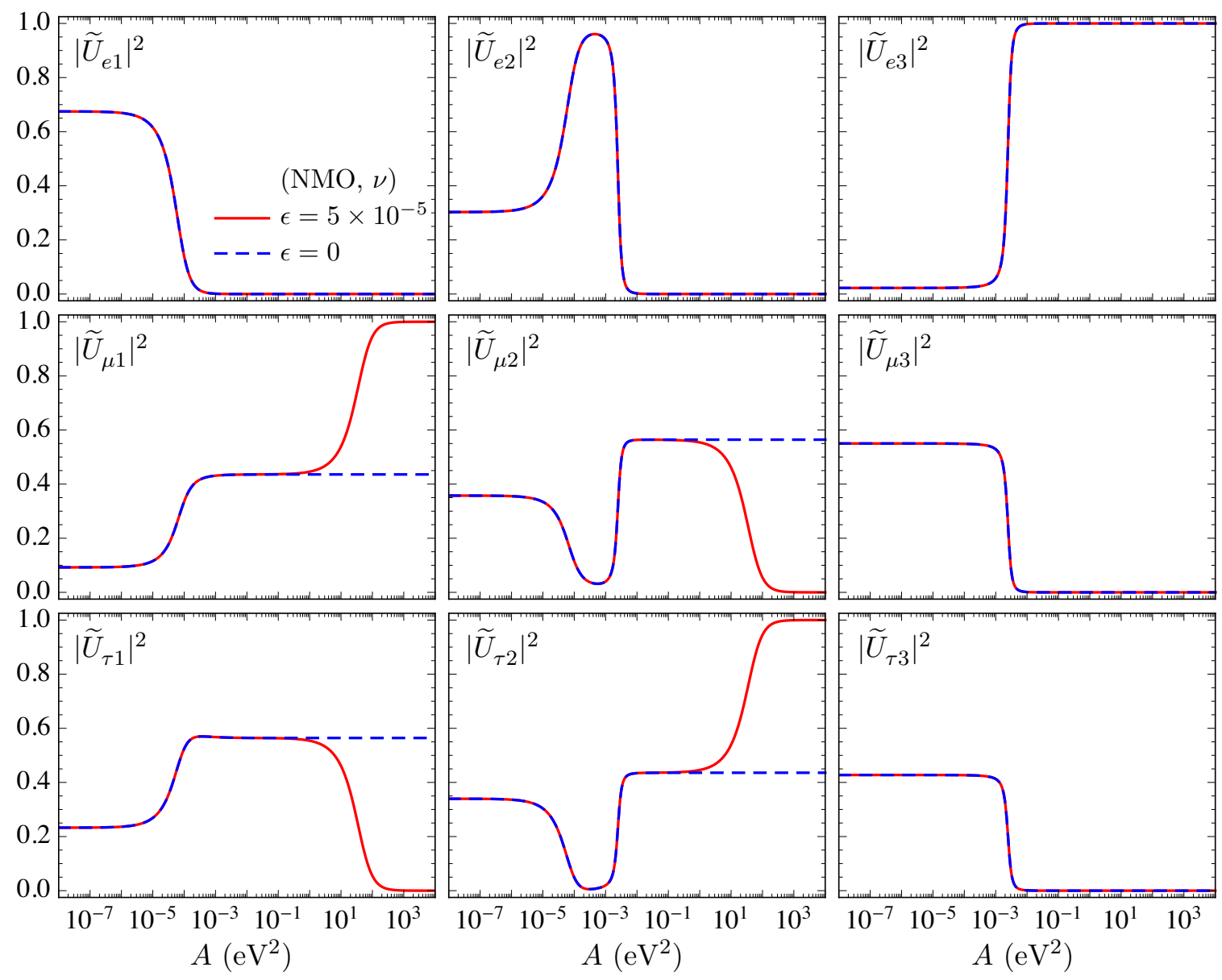

Figure 2. In the standard three-flavor mixing scheme, the illustration of how $\left|\widetilde{U}_{\alpha i}\right|^{2}$ (for $\alpha=e$, $\mu, \tau$ and $i=1,2,3)$ evolve with the matter effect parameter $A$ in the case (NMO, $\nu$ ) with or without radiative corrections, where the best-fit values of $\left(\theta_{12}, \theta_{13}, \theta_{23}, \delta, \Delta_{21}, \Delta_{31}\right)$ in refs. [36, 37] have been input.

where $\theta \in[0, \pi / 2]$ and

$$
\tan ^{2} \theta=\frac{\left|\widetilde{U}_{\mu 2}\right|^{2}}{\left|\widetilde{U}_{\mu 1}\right|^{2}} \simeq \frac{\xi-A \epsilon-\Delta_{21}\left(\left|U_{\tau 2}\right|^{2}-\left|U_{\mu 2}\right|^{2}\right)-\Delta_{31}\left(\left|U_{\tau 3}\right|^{2}-\left|U_{\mu 3}\right|^{2}\right)}{\xi+A \epsilon+\Delta_{21}\left(\left|U_{\tau 2}\right|^{2}-\left|U_{\mu 2}\right|^{2}\right)+\Delta_{31}\left(\left|U_{\tau 3}\right|^{2}-\left|U_{\mu 3}\right|^{2}\right)}
$$

Similarly, the neutrino oscillation probability $\widetilde{P}_{\alpha \beta}$ in eq. (2.11) can be approximately written as

$$
\begin{aligned}
& \widetilde{P}_{e e} \simeq 1, \quad \widetilde{P}_{e \mu} \simeq 0, \quad \widetilde{P}_{e \tau} \simeq 0, \\
& \widetilde{P}_{\mu e} \simeq 0, \quad \widetilde{P}_{\mu \mu} \simeq 1-\sin ^{2} 2 \theta \sin ^{2} \frac{\widetilde{\Delta}_{21} L}{4 E}, \quad \widetilde{P}_{\mu \tau} \simeq \sin ^{2} 2 \theta \sin ^{2} \frac{\widetilde{\Delta}_{21} L}{4 E}, \\
& \widetilde{P}_{\tau e} \simeq 0, \quad \widetilde{P}_{\tau \mu} \simeq \sin ^{2} 2 \theta \sin ^{2} \frac{\widetilde{\Delta}_{21} L}{4 E}, \quad \widetilde{P}_{\tau \tau} \simeq 1-\sin ^{2} 2 \theta \sin ^{2} \frac{\widetilde{\Delta}_{21} L}{4 E},
\end{aligned}
$$

where $\widetilde{\Delta}_{21}$ is taken from eq. $(2.13)$ and $\sin ^{2} 2 \theta=4\left|\widetilde{U}_{\mu 1}\right|^{2}\left(1-\left|\widetilde{U}_{\mu 1}\right|^{2}\right)$ with $\left|\widetilde{U}_{\mu 1}\right|^{2}$ being taken from eq. (2.15). Note that eq. (2.18) is similar to eq. (2.4) in ref. [25] except that we include 


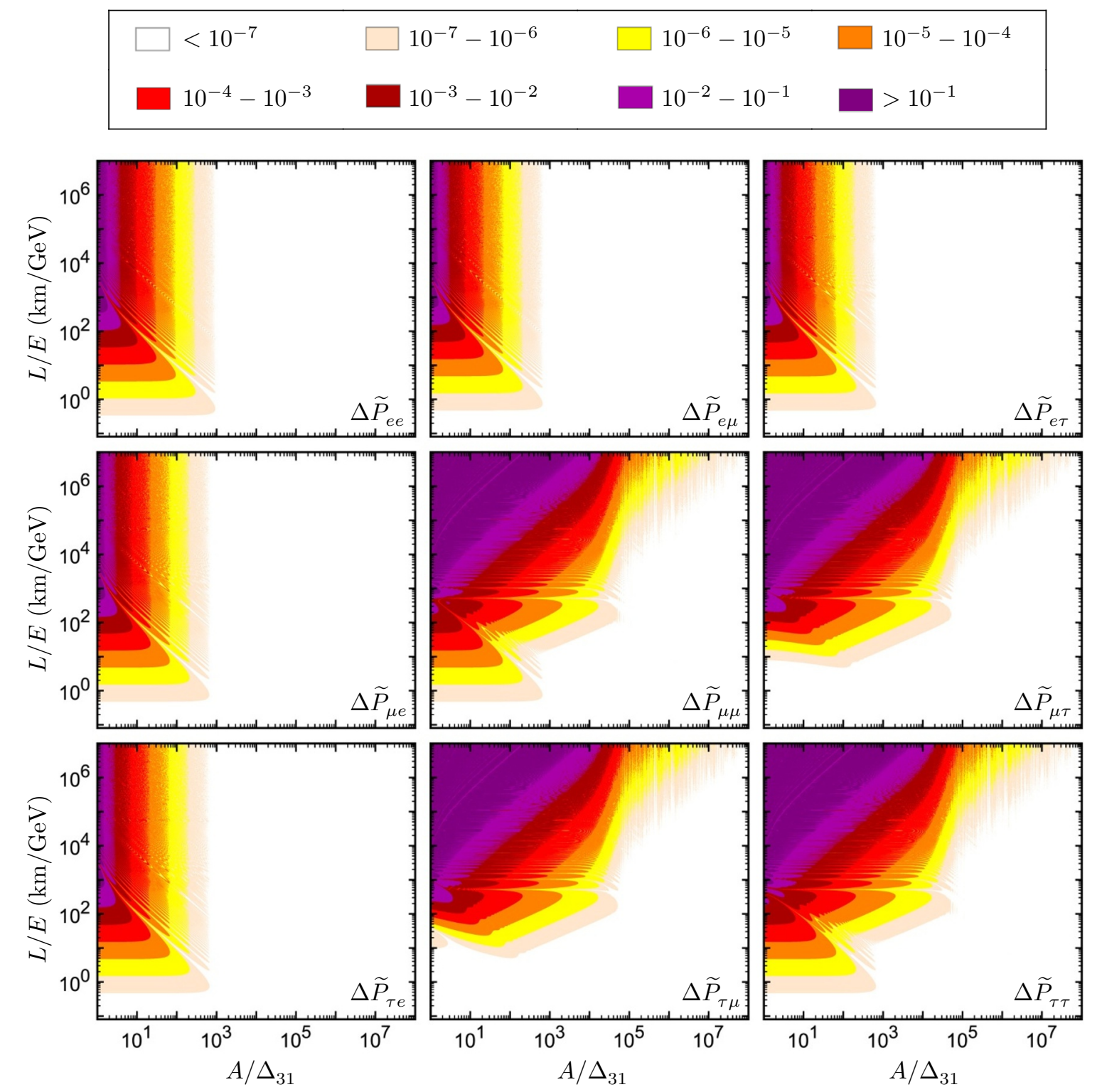

Figure 3. The absolute errors of our analytical approximations in eq. (2.18), where the best-fit values of $\left(\theta_{12}, \theta_{13}, \theta_{23}, \delta, \Delta_{21}, \Delta_{31}\right)$ in ref. [36, 37] and $\epsilon \simeq 5 \times 10^{-5}$ [26] have been input.

radiative corrections in $\theta$ and $\widetilde{\Delta}_{21}$. In order to numerically test the accuracies, we define the absolute error of $\widetilde{P}_{\alpha \beta}$ as $\Delta \widetilde{P}_{\alpha \beta}=\left|\left(\widetilde{P}_{\alpha \beta}\right)_{\text {Exact }}-\left(\widetilde{P}_{\alpha \beta}\right)_{\text {Approximate }}\right|$, where $\left(\widetilde{P}_{\alpha \beta}\right)_{\text {Exact }}$ stand for the exact results of $\widetilde{P}_{\alpha \beta}$ and $\left(\widetilde{P}_{\alpha \beta}\right)_{\text {Approximate }}$ represent the approximate results of $\widetilde{P}_{\alpha \beta}$. The absolute errors of $\widetilde{P}_{\alpha \beta}$ in eq. (2.18) with different $L / E$ and $A / \Delta_{31}$ are demonstrated in figure 3. Similar to the case without radiative corrections discussed in ref. [25], the analytical expressions of $\widetilde{P}_{\alpha \beta}$ in eq. (2.18) are accurate enough in most of the parameter space. For the upper left part in each subgraph of figure 3, we need to keep higher orders of $\Delta_{21} / A, \Delta_{31} / A$ and $\epsilon$, or just make perturbative expansions in terms of $\Delta_{21} / A$ and $\epsilon$ to improve the accuracies of $\widetilde{P}_{\alpha \beta}$. 


\begin{tabular}{|c|c|c|c|c|}
\hline & $(\mathrm{NMO}, \nu)$ & $(\mathrm{IMO}, \nu)$ & $(\mathrm{NMO}, \bar{\nu})$ & $(\mathrm{IMO}, \bar{\nu})$ \\
\hline$\widetilde{\Delta}_{21}$ & $A \epsilon$ & $A(1-\epsilon)$ & $A(1-\epsilon)$ & $A \epsilon$ \\
\hline$\widetilde{\Delta}_{31}$ & $A$ & $-A \epsilon$ & $A$ & $-A(1-\epsilon)$ \\
\hline$\widetilde{U}$ & $\left(\begin{array}{lll}0 & 0 & 1 \\
1 & 0 & 0 \\
0 & 1 & 0\end{array}\right)$ & $\left(\begin{array}{lll}0 & 1 & 0 \\
0 & 0 & 1 \\
1 & 0 & 0\end{array}\right)$ & $\left(\begin{array}{lll}1 & 0 & 0 \\
0 & 0 & 1 \\
0 & 1 & 0\end{array}\right)$ & $\left(\begin{array}{lll}0 & 0 & 1 \\
0 & 1 & 0 \\
1 & 0 & 0\end{array}\right)$ \\
\hline
\end{tabular}

Table 1. In the standard three-flavor mixing scheme, the analytical expressions of $\widetilde{\Delta}_{i j}$ (for $i j=$ $21,31)$ and $\widetilde{U}$ in the $A \rightarrow \infty$ limit, where we only show the terms of order $\mathcal{O}(A)$ for $\widetilde{\Delta}_{i j}$.

To be more explicit, if the $A \epsilon$ term is not bigger than the $\Delta_{21}$ term in eq. (2.15), we can further simplify $\left|\widetilde{U}_{\alpha i}\right|^{2}$ (for $\left.\alpha i=\mu 1, \mu 2, \tau 1, \tau 2\right)$ as $\left|\widetilde{U}_{\mu 1}\right|^{2} \simeq\left|\widetilde{U}_{\tau 2}\right|^{2} \simeq\left|U_{\tau 3}\right|^{2} /\left(\left|U_{\mu 3}\right|^{2}+\left|U_{\tau 3}\right|^{2}\right)$ and $\left|\widetilde{U}_{\mu 2}\right|^{2} \simeq\left|\widetilde{U}_{\tau 1}\right|^{2} \simeq\left|U_{\mu 3}\right|^{2} /\left(\left|U_{\mu 3}\right|^{2}+\left|U_{\tau 3}\right|^{2}\right)$. This is equivalent to the asymptotic values of $\left|\widetilde{U}_{\alpha i}\right|^{2}$ (for $\alpha i=\mu 1, \mu 2, \tau 1, \tau 2$ ) in the $A \rightarrow \infty$ limit when radiative corrections are not taken into account (the blue dashed line in figure 2). As the increase of $A$, the $A \epsilon$ term in eq. (2.15) becomes non-negligible. If the $A \epsilon$ term and $\Delta_{31}$ term are of the same order, the relation

$$
\frac{\mathrm{d}\left(\left|\widetilde{U}_{\mu 1}\right|^{2}\right)}{\mathrm{d} A} \simeq-\frac{\mathrm{d}\left(\left|\widetilde{U}_{\mu 2}\right|^{2}\right)}{\mathrm{d} A} \simeq \frac{2\left(1-\left|U_{\mu 3}\right|^{2}\right)\left|U_{\mu 3}\right|^{2} \epsilon}{\Delta_{31}},
$$

can be derived. This means $\theta=\arctan \left(\left|\widetilde{U}_{\mu 2}\right| /\left|\widetilde{U}_{\mu 1}\right|\right)$ will decrease with the increase of $A$ due to the existence of the radiative correction parameter $\epsilon$. In the $A \rightarrow \infty$ limit, it is easy to infer from eqs. (2.15) and (2.17) that $\left|\widetilde{U}_{\text {ai }}\right|^{2}$ trivially take 0 or 1 and $\theta$ is approaching zero, implying that all the three flavors do not oscillate into one another. Thus it makes no sense to discuss lepton flavor mixing in this extreme case. Considering the four cases $(\mathrm{NMO}, \nu),(\mathrm{IMO}, \nu),(\mathrm{IMO}, \bar{\nu})$ and $(\mathrm{IMO}, \bar{\nu})$ separately, we summarize the corresponding analytical expressions of $\widetilde{\Delta}_{i j}$ (for $i j=21,31$ ) and $\widetilde{U}$ in the $A \rightarrow \infty$ limit in table 1 while the other three cases will be discussed later.

\section{$2.2(\mathrm{IMO}, \nu)$}

Given a neutrino beam with inverted mass ordering, the evolution of $\widetilde{\Delta}_{i j}$ and $\left|\widetilde{U}_{\alpha i}\right|^{2}$ with $A$ are illustrated in the lower left panel of figure 1 and figure 4 , respectively. Note that there is no intersections between $\widetilde{\Delta}_{21}$ and $\widetilde{\Delta}_{31}$ in cases (IMO, $\nu$ ) and (IMO, $\bar{\nu}$ ) in figure 1 with $\left|\widetilde{\Delta}_{31}\right|=-\widetilde{\Delta}_{31}$ being shown in fact. In the case (IMO, $\nu$ ), we also note that $\widetilde{\Delta}_{21}>\left|\widetilde{\Delta}_{31}\right|$ holds when the matter effect parameter $A$ is big enough. Analytically, expanding eq. (2.2) in $\Delta_{21} / A, \Delta_{31} / A$ and $\epsilon$ directly leads to

$$
\begin{aligned}
& \widetilde{\Delta}_{21} \simeq A-\frac{1}{2}\left[A \epsilon+\left(1-3\left|U_{e 2}\right|^{2}\right) \Delta_{21}+\left(1-3\left|U_{e 3}\right|^{2}\right) \Delta_{31}+\xi\right], \\
& \widetilde{\Delta}_{31} \simeq-\xi
\end{aligned}
$$

with $\xi$ being defined in eq. (2.14). Consistent with figure $1, \widetilde{\Delta}_{31}$ approaches $-A \epsilon$ instead of a constant value in the $A \rightarrow \infty$ limit. To understand the asymptotic behaviors of $\left|\widetilde{U}_{\alpha i}\right|^{2}$ 

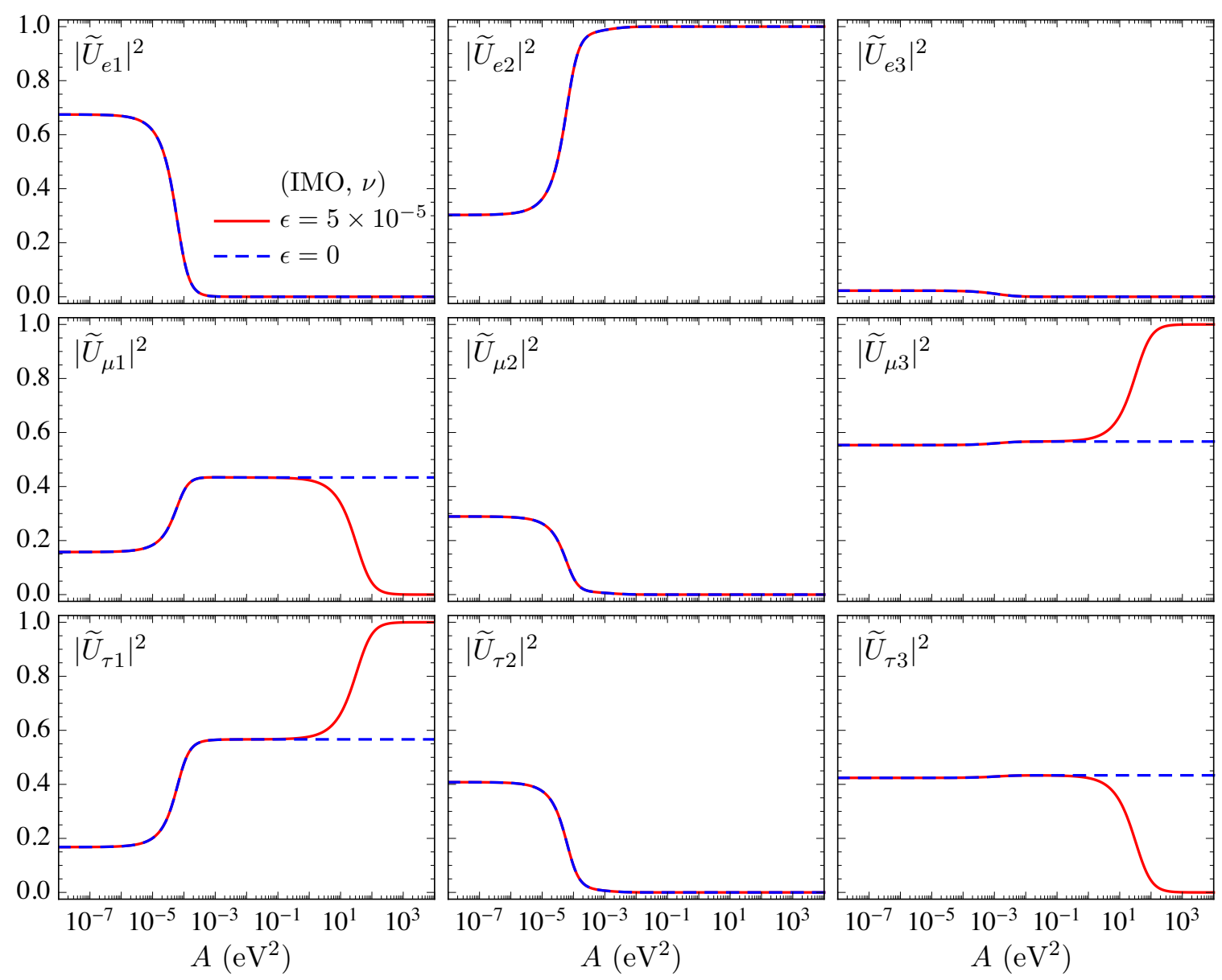

Figure 4. In the standard three-flavor mixing scheme, the illustration of how $\left|\widetilde{U}_{\alpha i}\right|^{2}$ (for $\alpha=$ $e, \mu, \tau$ and $i=1,2,3)$ evolve with the matter effect parameter $A$ in the case (IMO, $\nu$ ) with or without radiative corrections, where the best-fit values of $\left(\theta_{12}, \theta_{13}, \theta_{23}, \delta, \Delta_{21}, \Delta_{31}\right)$ in ref. [36, 37] have been input.

in the $A \rightarrow \infty$ limit shown in figure 4, we expand eq. (2.6) and get

$$
\begin{aligned}
& \left|\widetilde{U}_{e 1}\right|^{2} \simeq\left|\widetilde{U}_{e 3}\right|^{2} \simeq\left|\widetilde{U}_{\mu 2}\right|^{2} \simeq\left|\widetilde{U}_{\tau 2}\right|^{2} \simeq 0, \quad\left|\widetilde{U}_{e 2}\right|^{2} \simeq 1 \\
& \left|\widetilde{U}_{\mu 1}\right|^{2} \simeq\left|\widetilde{U}_{\tau 3}\right|^{2} \simeq \frac{1}{2}-\frac{A \epsilon+\Delta_{21}\left(\left|U_{\tau 2}\right|^{2}-\left|U_{\mu 2}\right|^{2}\right)+\Delta_{31}\left(\left|U_{\tau 3}\right|^{2}-\left|U_{\mu 3}\right|^{2}\right)}{2 \xi} \\
& \left|\widetilde{U}_{\mu 3}\right|^{2} \simeq\left|\widetilde{U}_{\tau 1}\right|^{2} \simeq \frac{1}{2}+\frac{A \epsilon+\Delta_{21}\left(\left|U_{\tau 2}\right|^{2}-\left|U_{\mu 2}\right|^{2}\right)+\Delta_{31}\left(\left|U_{\tau 3}\right|^{2}-\left|U_{\mu 3}\right|^{2}\right)}{2 \xi}
\end{aligned}
$$

So one can use only one parameter to approximately describe lepton flavor mixing,

$$
\left.\widetilde{U}\right|_{A \gg \Delta_{31}} \simeq\left(\begin{array}{ccc}
0 & 1 & 0 \\
\cos \theta & 0 & \sin \theta \\
-\sin \theta & 0 & \cos \theta
\end{array}\right)
$$


where $\theta \in[0, \pi / 2]$ and

$$
\tan ^{2} \theta=\frac{\left|\widetilde{U}_{\mu 3}\right|^{2}}{\left|\widetilde{U}_{\mu 1}\right|^{2}} \simeq \frac{\xi+A \epsilon+\Delta_{21}\left(\left|U_{\tau 2}\right|^{2}-\left|U_{\mu 2}\right|^{2}\right)+\Delta_{31}\left(\left|U_{\tau 3}\right|^{2}-\left|U_{\mu 3}\right|^{2}\right)}{\xi-A \epsilon-\Delta_{21}\left(\left|U_{\tau 2}\right|^{2}-\left|U_{\mu 2}\right|^{2}\right)-\Delta_{31}\left(\left|U_{\tau 3}\right|^{2}-\left|U_{\mu 3}\right|^{2}\right)} .
$$

The analytical approximations of $\widetilde{P}_{\alpha \beta}$ in eq. (2.11) turn out to be

$$
\begin{aligned}
& \widetilde{P}_{e e} \simeq 1, \quad \widetilde{P}_{e \mu} \simeq 0, \quad \widetilde{P}_{e \tau} \simeq 0, \\
& \widetilde{P}_{\mu e} \simeq 0, \quad \widetilde{P}_{\mu \mu} \simeq 1-\sin ^{2} 2 \theta \sin ^{2} \frac{\widetilde{\Delta}_{31} L}{4 E}, \quad \widetilde{P}_{\mu \tau} \simeq \sin ^{2} 2 \theta \sin ^{2} \frac{\widetilde{\Delta}_{31} L}{4 E}, \\
& \widetilde{P}_{\tau e} \simeq 0, \quad \widetilde{P}_{\tau \mu} \simeq \sin ^{2} 2 \theta \sin ^{2} \frac{\widetilde{\Delta}_{31} L}{4 E}, \quad \widetilde{P}_{\tau \tau} \simeq 1-\sin ^{2} 2 \theta \sin ^{2} \frac{\widetilde{\Delta}_{31} L}{4 E},
\end{aligned}
$$

where $\widetilde{\Delta}_{31}$ comes from eq. (2.20) and $\sin ^{2} 2 \theta=4\left|\widetilde{U}_{\mu 1}\right|^{2}\left(1-\left|\widetilde{U}_{\mu 1}\right|^{2}\right)$ with $\left|\widetilde{U}_{\mu 1}\right|^{2}$ coming from eq. (2.21). For simplicity, we do not show the accuracies of eq. (2.24), which are very similar to the case (NMO, $\nu$ ) in figure 3. Comparing eq. (2.24) with eq. (2.18), we find that it is impossible to discriminate the normal mass ordering from the inverted mass ordering from neutrino oscillations if the matter density is very big.

We also notice that if the $\Delta_{21}$ term and $A \epsilon$ term in eq. (2.21) are of the same order, one can omit them and get $\left|\widetilde{U}_{\mu 1}\right|^{2} \simeq\left|\widetilde{U}_{\tau 3}\right|^{2} \simeq\left|U_{\tau 3}\right|^{2} /\left(\left|U_{\mu 3}\right|^{2}+\left|U_{\tau 3}\right|^{2}\right)$ and $\left|\widetilde{U}_{\mu 3}\right|^{2} \simeq$ $\left|\widetilde{U}_{\tau 1}\right|^{2} \simeq\left|U_{\mu 3}\right|^{2} /\left(\left|U_{\mu 3}\right|^{2}+\left|U_{\tau 3}\right|^{2}\right)$. This corresponds to the fixed values of $\left|\widetilde{U}_{\alpha i}\right|^{2}$ (for $\alpha i=\mu 1, \mu 3, \tau 1, \tau 3)$ in the $A \rightarrow \infty$ limit if radiative corrections are not taken into account (the blue dashed line in figure 4). As the increase of $A$, the $A \epsilon$ term will gradually dominate and the neutrino oscillation behaviors can be very sensitive to $A$. In the limit of $A \rightarrow \infty, \theta$ approaches $\pi / 2$ and there will be no neutrino oscillation phenomenon.

\section{$2.3(\mathrm{NMO}, \bar{\nu})$}

Considering an antineutrino beam with normal mass ordering, we make the replacements $A \rightarrow-A$ and $U \rightarrow-U^{*}$ in eqs. (2.1) and (2.6), and draw the corresponding evolution of $\widetilde{\Delta}_{i j}$ and $\left|\widetilde{U}_{\alpha i}\right|^{2}$ with $\mathrm{A}$ in the upper right panel of figure 1 and figure 5 , respectively. Note that we always have $\widetilde{\Delta}_{31}>\widetilde{\Delta}_{21}$ in this case although the difference between them is too small to be shown clearly in figure 1 if $A$ is big enough. The radiative corrections to both $\widetilde{\Delta}_{21}$ and $\widetilde{\Delta}_{31}$ are very small, which can be analytically understood. By performing perturbative expansions, $\widetilde{\Delta}_{i j}$ (for $i j=21,31$ ) in eq. (2.1) are reduced to

$$
\begin{aligned}
& \widetilde{\Delta}_{21} \simeq A-\frac{1}{2}\left[A \epsilon-\left(1-3\left|U_{e 2}\right|^{2}\right) \Delta_{21}-\left(1-3\left|U_{e 3}\right|^{2}\right) \Delta_{31}+\xi\right], \\
& \widetilde{\Delta}_{31} \simeq A-\frac{1}{2}\left[A \epsilon-\left(1-3\left|U_{e 2}\right|^{2}\right) \Delta_{21}-\left(1-3\left|U_{e 3}\right|^{2}\right) \Delta_{31}-\xi\right]
\end{aligned}
$$

with

$$
\begin{aligned}
\xi=\{ & {\left[\left(1-\left|U_{e 2}\right|^{2}\right) \Delta_{21}+\left(1-\left|U_{e 3}\right|^{2}\right) \Delta_{31}-A \epsilon\right]^{2}+4 A \epsilon\left(\left|U_{\mu 2}\right|^{2} \Delta_{21}+\left|U_{\mu 3}\right|^{2} \Delta_{31}\right) } \\
& \left.-4\left|U_{e 1}\right|^{2} \Delta_{21} \Delta_{31}\right\}^{1 / 2} .
\end{aligned}
$$



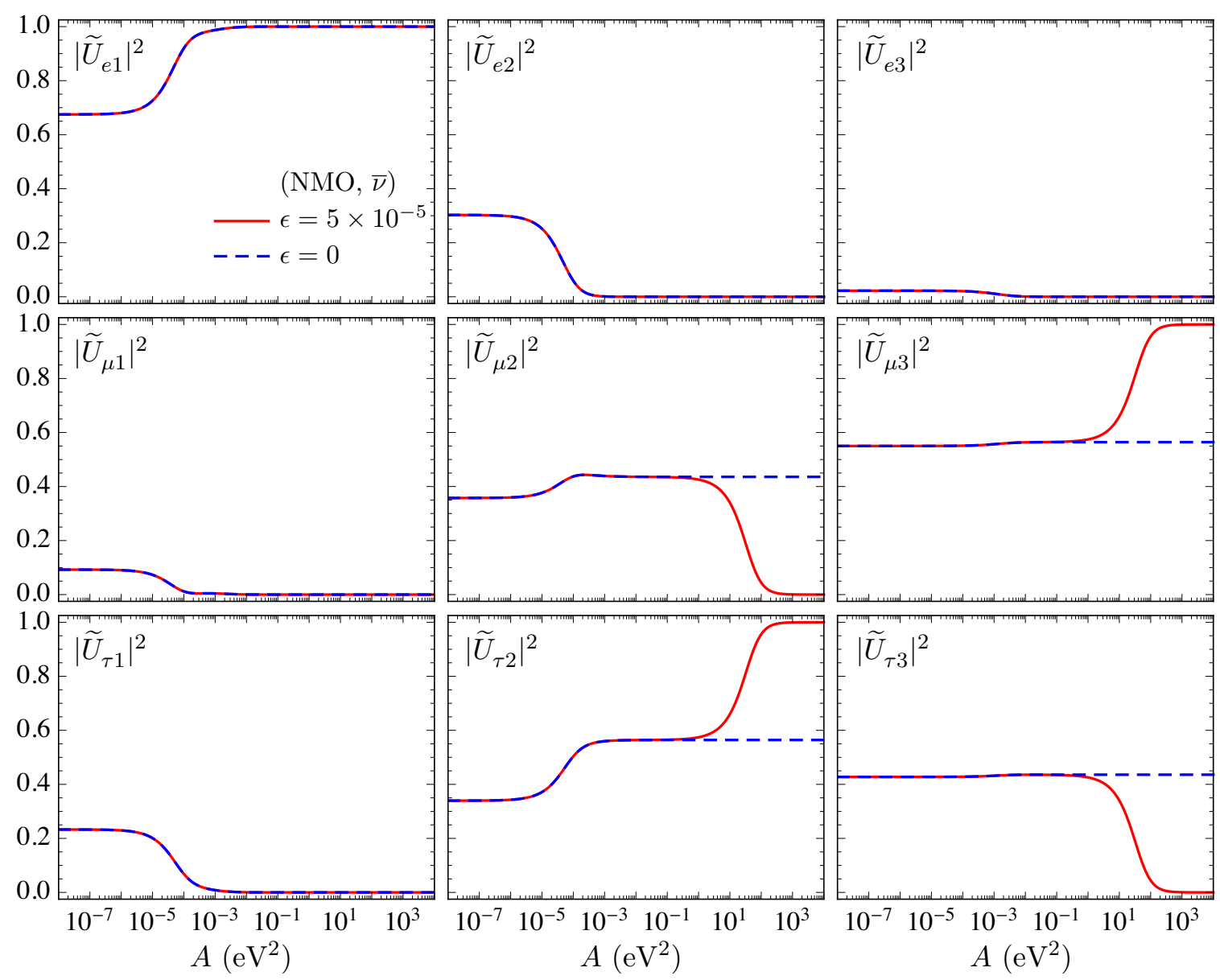

Figure 5. In the standard three-flavor mixing scheme, the illustration of how $\left|\widetilde{U}_{\alpha i}\right|^{2}$ (for $\alpha=e$, $\mu, \tau$ and $i=1,2,3)$ evolve with the matter effect parameter $A$ in the case (NMO, $\bar{\nu}$ ) with or without radiative corrections, where the best-fit values of $\left(\theta_{12}, \theta_{13}, \theta_{23}, \delta, \Delta_{21}, \Delta_{31}\right)$ in refs. [36, 37] have been input.

From eq. (2.25), it is clear that the leading order of $\widetilde{\Delta}_{21}$ and $\widetilde{\Delta}_{31}$ is $A$ and the radiative corrections in the next-to-leading order do not matter a lot. The only difference between eq. (2.26) (the expression of $\xi$ in the case $(\mathrm{NMO}, \bar{\nu})$ ) and eq. (2.14) (the expression of $\xi$ in the case $(\mathrm{NMO}, \nu))$ is the sign of $\epsilon$. Similarly, $\left|\widetilde{U}_{\alpha i}\right|^{2}$ can be expanded as

$$
\begin{aligned}
& \left|\widetilde{U}_{e 2}\right|^{2} \simeq\left|\widetilde{U}_{e 3}\right|^{2} \simeq\left|\widetilde{U}_{\mu 1}\right|^{2} \simeq\left|\widetilde{U}_{\tau 1}\right|^{2} \simeq 0, \quad\left|\widetilde{U}_{e 1}\right|^{2} \simeq 1 \\
& \left|\widetilde{U}_{\mu 2}\right|^{2} \simeq\left|\widetilde{U}_{\tau 3}\right|^{2} \simeq \frac{1}{2}-\frac{A \epsilon-\Delta_{21}\left(\left|U_{\tau 2}\right|^{2}-\left|U_{\mu 2}\right|^{2}\right)-\Delta_{31}\left(\left|U_{\tau 3}\right|^{2}-\left|U_{\mu 3}\right|^{2}\right)}{2 \xi} \\
& \left|\widetilde{U}_{\mu 3}\right|^{2} \simeq\left|\widetilde{U}_{\tau 2}\right|^{2} \simeq \frac{1}{2}+\frac{A \epsilon-\Delta_{21}\left(\left|U_{\tau 2}\right|^{2}-\left|U_{\mu 2}\right|^{2}\right)-\Delta_{31}\left(\left|U_{\tau 3}\right|^{2}-\left|U_{\mu 3}\right|^{2}\right)}{2 \xi}
\end{aligned}
$$

namely,

$$
\left.\widetilde{U}\right|_{A \gg \Delta_{31}} \simeq\left(\begin{array}{ccc}
1 & 0 & 0 \\
0 & \cos \theta & \sin \theta \\
0 & -\sin \theta & \cos \theta
\end{array}\right)
$$


where $\theta \in[0, \pi / 2]$ and

$$
\tan ^{2} \theta=\frac{\left|\widetilde{U}_{\mu 3}\right|^{2}}{\left|\widetilde{U}_{\mu 2}\right|^{2}} \simeq \frac{\xi+A \epsilon-\Delta_{21}\left(\left|U_{\tau 2}\right|^{2}-\left|U_{\mu 2}\right|^{2}\right)-\Delta_{31}\left(\left|U_{\tau 3}\right|^{2}-\left|U_{\mu 3}\right|^{2}\right)}{\xi-A \epsilon+\Delta_{21}\left(\left|U_{\tau 2}\right|^{2}-\left|U_{\mu 2}\right|^{2}\right)+\Delta_{31}\left(\left|U_{\tau 3}\right|^{2}-\left|U_{\mu 3}\right|^{2}\right)} .
$$

The corresponding neutrino oscillation probabilities in matter are approximately

$$
\begin{array}{lll}
\widetilde{P}_{e e} \simeq 1, & \widetilde{P}_{e \mu} \simeq 0, & \widetilde{P}_{e \tau} \simeq 0, \\
\widetilde{P}_{\mu e} \simeq 0, & \widetilde{P}_{\mu \mu} \simeq 1-\sin ^{2} 2 \theta \sin ^{2} \frac{\widetilde{\Delta}_{32} L}{4 E}, & \widetilde{P}_{\mu \tau} \simeq \sin ^{2} 2 \theta \sin ^{2} \frac{\widetilde{\Delta}_{32} L}{4 E}, \\
\widetilde{P}_{\tau e} \simeq 0, & \widetilde{P}_{\tau \mu} \simeq \sin ^{2} 2 \theta \sin ^{2} \frac{\widetilde{\Delta}_{32} L}{4 E}, & \widetilde{P}_{\tau \tau} \simeq 1-\sin ^{2} 2 \theta \sin ^{2} \frac{\widetilde{\Delta}_{32} L}{4 E},
\end{array}
$$

where $\sin ^{2} 2 \theta=\left|\widetilde{U}_{\mu 2}\right|^{2}\left(1-\left|\widetilde{U}_{\mu 2}\right|^{2}\right)$ and $\widetilde{\Delta}_{32} \simeq \xi$ from eq. (2.25). The accuracies of $\widetilde{P}_{\alpha \beta}$ in eq. (2.30) are similar to the case (NMO, $\nu)$ in figure 3 .

If $A$ is small enough, we can ignore the smaller terms of $\Delta_{21}$ and $A \epsilon$ in eq. (2.27), and obtain $\left|\widetilde{U}_{\mu 2}\right|^{2} \simeq\left|\widetilde{U}_{\tau 3}\right|^{2} \simeq\left|U_{\tau 3}\right|^{2} /\left(\left|U_{\mu 3}\right|^{2}+\left|U_{\tau 3}\right|^{2}\right)$ and $\left|\widetilde{U}_{\mu 3}\right|^{2} \simeq\left|\widetilde{U}_{\tau 2}\right|^{2} \simeq\left|U_{\mu 3}\right|^{2} /\left(\left|U_{\mu 3}\right|^{2}+\right.$ $\left|U_{\tau 3}\right|^{2}$ ). This is consistent with the fixed values of $\left|\widetilde{U}_{\alpha i}\right|^{2}$ (for $\alpha i=\mu 2, \mu 3, \tau 2, \tau 3$ ) in the $A \rightarrow \infty$ limit if radiative corrections are not included (the blue dashed line in figure 5). If the term $A \epsilon$ too big to be abandoned, the neutrino flavor mixing can be significantly affected by $A$. In the $A \rightarrow \infty$ limit, $\left|\widetilde{U}_{\alpha i}\right|^{2}$ trivially take 0 or 1 and $\theta$ approaches $\pi / 2$, leading to no neutrino oscillations.

\section{$2.4(\mathrm{IMO}, \bar{\nu})$}

Similarly, in the case (IMO, $\bar{\nu})$, i.e. an antineutrino beam with inverted mass ordering, we illustrate the evolution of $\widetilde{\Delta}_{i j}$ and $\left|\widetilde{U}_{\alpha i}\right|^{2}$ in the lower right panel of figure 1 and figure 6 , respectively. Through making perturbative expansions, $\widetilde{\Delta}_{i j}$ and $\left|\widetilde{U}_{\alpha i}\right|^{2}$ can be approximately expressed as

$$
\begin{aligned}
& \widetilde{\Delta}_{21} \simeq \xi \\
& \widetilde{\Delta}_{31} \simeq-A+\frac{1}{2}\left[A \epsilon-\left(1-3\left|U_{e 2}\right|^{2}\right) \Delta_{21}-\left(1-3\left|U_{e 3}\right|^{2}\right) \Delta_{31}+\xi\right],
\end{aligned}
$$

and

$$
\begin{aligned}
& \left|\widetilde{U}_{e 1}\right|^{2} \simeq\left|\widetilde{U}_{e 2}\right|^{2} \simeq\left|\widetilde{U}_{\mu 3}\right|^{2} \simeq\left|\widetilde{U}_{\tau 3}\right|^{2} \simeq 0, \quad\left|\widetilde{U}_{e 3}\right|^{2} \simeq 1 \\
& \left|\widetilde{U}_{\mu 1}\right|^{2} \simeq\left|\widetilde{U}_{\tau 2}\right|^{2} \simeq \frac{1}{2}-\frac{A \epsilon-\Delta_{21}\left(\left|U_{\tau 2}\right|^{2}-\left|U_{\mu 2}\right|^{2}\right)-\Delta_{31}\left(\left|U_{\tau 3}\right|^{2}-\left|U_{\mu 3}\right|^{2}\right)}{2 \xi} \\
& \left|\widetilde{U}_{\mu 2}\right|^{2} \simeq\left|\widetilde{U}_{\tau 1}\right|^{2} \simeq \frac{1}{2}+\frac{A \epsilon-\Delta_{21}\left(\left|U_{\tau 2}\right|^{2}-\left|U_{\mu 2}\right|^{2}\right)-\Delta_{31}\left(\left|U_{\tau 3}\right|^{2}-\left|U_{\mu 3}\right|^{2}\right)}{2 \xi}
\end{aligned}
$$

where $\xi$ has been defined in eq. (2.26). From either figure 1 or eq. (2.31), it is clear that the radiative corrections to $\widetilde{\Delta}_{21}$ are very important in this case if the matter density is big 


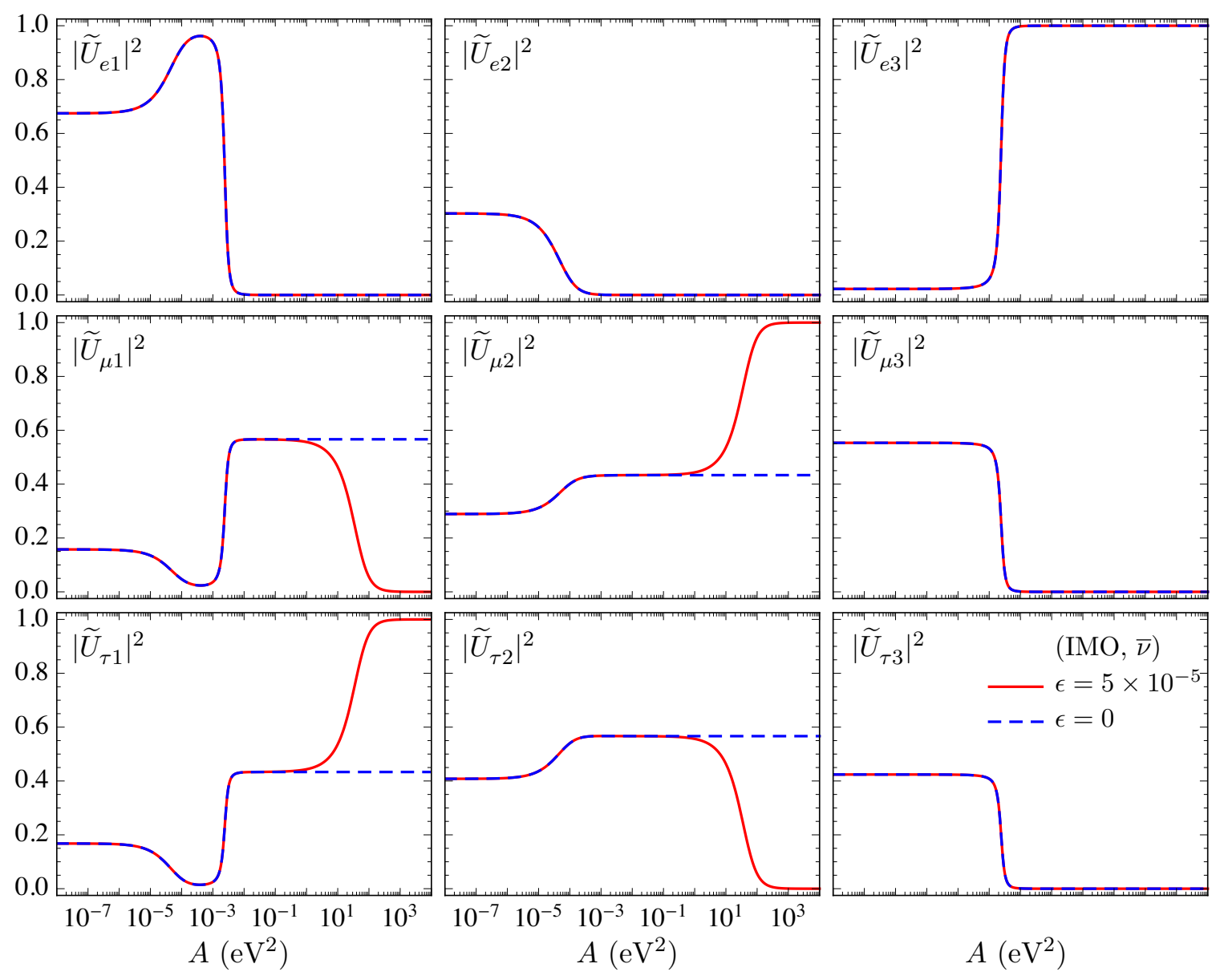

Figure 6. In the standard three-flavor mixing scheme, the illustration of how $\left|\widetilde{U}_{\alpha i}\right|^{2}$ (for $\alpha=e$, $\mu, \tau$ and $i=1,2,3)$ evolve with the matter effect parameter $A$ in the case (IMO, $\bar{\nu}$ ) with or without radiative corrections, where the best-fit values of $\left(\theta_{12}, \theta_{13}, \theta_{23}, \delta, \Delta_{21}, \Delta_{31}\right)$ in refs. [36, 37] have been input.

enough. According to eq. (2.32), the lepton flavor mixing matrix can be approximately parametrized as:

$$
\left.\widetilde{U}\right|_{A \gg \Delta_{31}} \simeq\left(\begin{array}{ccc}
0 & 0 & 1 \\
\cos \theta & \sin \theta & 0 \\
-\sin \theta & \cos \theta & 0
\end{array}\right)
$$

where $\theta \in[0, \pi / 2]$ and

$$
\tan ^{2} \theta \simeq \frac{\xi+A \epsilon-\Delta_{21}\left(\left|U_{\tau 2}\right|^{2}-\left|U_{\mu 2}\right|^{2}\right)-\Delta_{31}\left(\left|U_{\tau 3}\right|^{2}-\left|U_{\mu 3}\right|^{2}\right)}{\xi-A \epsilon+\Delta_{21}\left(\left|U_{\tau 2}\right|^{2}-\left|U_{\mu 2}\right|^{2}\right)+\Delta_{31}\left(\left|U_{\tau 3}\right|^{2}-\left|U_{\mu 3}\right|^{2}\right)}
$$

The corresponding analytical approximations of $\widetilde{P}_{\alpha \beta}$ are the same as eq. (2.18) except that $\widetilde{\Delta}_{21}$ and $\sin ^{2} 2 \theta=\left|\widetilde{U}_{\mu 1}\right|^{2}\left(1-\left|\widetilde{U}_{\mu 1}\right|^{2}\right)$ should be taken from eq. (2.31) and eq. (2.32), respectively. Comparing eq. (2.18) and eq. (2.30), we find that it is impossible to discrimi- 
nate between the normal mass ordering and the inverted mass ordering from antineutrino oscillations if the matter density is very big.

If the $\Delta_{21}$ term and the $A \epsilon$ term in eq. (2.32) are of the same order, we can omit them and arrive at $\left|\widetilde{U}_{\mu 1}\right|^{2} \simeq\left|\widetilde{U}_{\tau 2}\right|^{2} \simeq\left|U_{\mu 3}\right|^{2} /\left(\left|U_{\mu 3}\right|^{2}+\left|U_{\tau 3}\right|^{2}\right)$ and $\left|\widetilde{U}_{\mu 2}\right|^{2} \simeq\left|\widetilde{U}_{\tau 1}\right|^{2} \simeq$ $\left|U_{\tau 3}\right|^{2} /\left(\left|U_{\mu 3}\right|^{2}+\left|U_{\tau 3}\right|^{2}\right)$. This coincides with the fixed values of $\left|\widetilde{U}_{\alpha i}\right|^{2}$ (for $\left.\alpha i=\mu 1, \mu 2, \tau 1, \tau 2\right)$ in the $A \rightarrow \infty$ limit if radiative corrections are not included (the blue dashed line in figure 6). If the term $A \epsilon$ is too big to be omitted, it will affect the neutrino flavor mixing a lot. In the $A \rightarrow \infty, \theta$ approaches $\pi / 2$ and no neutrino oscillations between $\nu_{e}, \nu_{\mu}$ and $\nu_{\tau}$ will happen.

\section{The $(3+1)$ flavor mixing scheme}

Now we turn to the $(3+1)$ flavor mixing scheme with one more light sterile neutrino. The corresponding Hamiltonian $\mathcal{H}_{\mathrm{m}}^{s}$ describing the propagation of neutrinos in matter has been shown in eq. (1.4). Analogous to ref. [38], the eigenvalues $\lambda$ of $\mathcal{H}_{\mathrm{m}}^{s}$ can be derived by solving the equation

$$
\lambda^{4}+b \lambda^{3}+c \lambda^{2}+d \lambda+e=0
$$

where the expressions of the relevant coefficients are as follows

$$
\begin{aligned}
b= & -\sum_{i} \Delta_{i 1}-A(1+\epsilon)-A^{\prime}, \\
c= & \sum_{i<j} \Delta_{i 1} \Delta_{j 1}+A \sum_{i} \Delta_{i 1}\left(1-\left|V_{e i}\right|^{2}\right)+\epsilon A \sum_{i} \Delta_{i 1}\left(1-\left|V_{\tau i}\right|^{2}\right) \\
& +A^{\prime} \sum_{i} \Delta_{i 1}\left(1-\left|V_{s i}\right|^{2}\right)+A A^{\prime}(1+\epsilon)+A^{2} \epsilon \\
d= & -\Delta_{21} \Delta_{31} \Delta_{41}-\sum_{i, j, k} \frac{\varepsilon_{i j k}^{2}}{2} \Delta_{i 1} \Delta_{j 1}\left(A\left|V_{e k}\right|^{2}+\epsilon A\left|V_{\tau k}\right|^{2}+A^{\prime}\left|V_{s k}^{2}\right|\right) \\
& -A A^{\prime} \sum_{i} \Delta_{i 1}\left(\left|V_{\mu i}\right|^{2}+\left|V_{\tau i}\right|^{2}\right)-\epsilon A^{2} \sum_{i} \Delta_{i 1}\left(\left|V_{\mu i}\right|^{2}+\left|V_{s i}\right|^{2}\right) \\
& -\epsilon A A^{\prime} \sum_{i} \Delta_{i 1}\left(\left|V_{e i}\right|^{2}+\left|V_{\mu i}\right|^{2}\right)-\epsilon A^{2} A^{\prime}, \\
e= & \Delta_{21} \Delta_{31} \Delta_{41}\left(A\left|V_{e 1}\right|^{2}+\epsilon A\left|V_{\tau 1}\right|^{2}+A^{\prime}\left|V_{s 1}\right|^{2}\right)+A A^{\prime} \sum_{i, j, k, l} \frac{\varepsilon_{i j k l}^{2}}{4} \Delta_{i 1} \Delta_{j 1} C_{e s}^{k l} \\
& +\epsilon A^{2} \sum_{i, j, k, l} \frac{\varepsilon_{i j k l}^{2}}{4} \Delta_{i 1} \Delta_{j 1} C_{e \tau}^{k l}+\epsilon A A^{\prime} \sum_{i, j, k, l} \frac{\varepsilon_{i j k l}^{2}}{4} \Delta_{i 1} \Delta_{j 1} C_{s \tau}^{k l}+\epsilon A^{2} A^{\prime} \sum_{i} \Delta_{i 1}\left|V_{\mu i}\right|^{2}
\end{aligned}
$$

with $i, j, k, l=1,2,3,4 ; \varepsilon_{i j k l}$ being four-dimension Levi-Civita symbol; and

$$
C_{\alpha \beta}^{i j}=\left|V_{\alpha i} V_{\beta j}-V_{\alpha j} V_{\beta i}\right|^{2}
$$

for $\alpha \beta=e s, e \tau, s \tau$. By defining $\lambda_{1}<\lambda_{2}<\lambda_{3}<\lambda_{4}$ for the normal mass ordering and $\lambda_{3}<\lambda_{1}<\lambda_{2}<\lambda_{4}$ for the inverted ordering, we have $\widetilde{\Delta}_{i j}=\lambda_{i}-\lambda_{j}$. After a tedious but 
straightforward calculation, the exact expressions of $\widetilde{\Delta}_{i j}$ (for $\left.i j=21,31,41\right)$ are [38]

$$
\begin{aligned}
& \widetilde{\Delta}_{21}=\sqrt{-4 S^{2}-2 p+\frac{q}{S}}, \\
& \widetilde{\Delta}_{31}=2 S+\frac{1}{2}\left(\sqrt{-4 S^{2}-2 p+\frac{q}{S}}-\sqrt{-4 S^{2}-2 p-\frac{q}{S}}\right), \\
& \widetilde{\Delta}_{41}=2 S+\frac{1}{2}\left(\sqrt{-4 S^{2}-2 p+\frac{q}{S}}+\sqrt{-4 S^{2}-2 p-\frac{q}{S}}\right)
\end{aligned}
$$

for the normal mass ordering $\left(m_{1}<m_{2}<m_{3}<m_{4}\right)$ and

$$
\begin{aligned}
& \widetilde{\Delta}_{21}=2 S-\frac{1}{2}\left(\sqrt{-4 S^{2}-2 p+\frac{q}{S}}+\sqrt{-4 S^{2}-2 p-\frac{q}{S}}\right), \\
& \widetilde{\Delta}_{31}=-\sqrt{-4 S^{2}-2 p+\frac{q}{S}}, \\
& \widetilde{\Delta}_{41}=2 S-\frac{1}{2}\left(\sqrt{-4 S^{2}-2 p+\frac{q}{S}}-\sqrt{-4 S^{2}-2 p-\frac{q}{S}}\right)
\end{aligned}
$$

for the inverted mass ordering $\left(m_{3}<m_{1}<m_{2}<m_{4}\right)$, where

$$
\begin{aligned}
p & =c-\frac{3 b^{2}}{8}, \quad q=\frac{b^{3}}{8}-\frac{1}{2} b c+d, \\
S & =\frac{1}{2} \sqrt{-\frac{2}{3} p+\frac{2}{3} \sqrt{c^{2}-3 b d+12 e} \cos \left\{\frac{1}{3}\left[\arccos \left(\frac{2 c^{3}-9 b c d+27 b^{2} e+27 d^{2}-72 c e}{2\left(c^{2}-3 b d+12 e\right)^{3 / 2}}\right)\right]\right\} .}
\end{aligned}
$$

Note that the formulas of $\widetilde{\Delta}_{i j}$ in eq. (3.4) are the same as eq. (3.4) in ref. [38] except that the coefficients $b, c, d$ and $e$ in eq. (3.2) include radiative corrections. By taking the trace of $\mathcal{H}_{\mathrm{m}}^{s}, B$ can be expressed as

$$
B=\frac{1}{4}\left[\Delta_{21}+\Delta_{31}+\Delta_{41}+A(1+\epsilon)+A^{\prime}-\widetilde{\Delta}_{21}-\widetilde{\Delta}_{31}-\widetilde{\Delta}_{41}\right] .
$$

By considering the unitarity conditions of $\widetilde{V}$ and the sum rules derived from $\mathcal{H}_{\mathrm{m}}^{s},\left(\mathcal{H}_{\mathrm{m}}^{s}\right)^{2}$ and $\left(\mathcal{H}_{\mathrm{m}}^{s}\right)^{3}$, we get a full set of linear equations of $\widetilde{V}_{\alpha i} \widetilde{V}_{\beta i}^{*}$ (for $i=1,2,3,4$ and $\alpha, \beta=e, \mu, \tau, s$ ),

$$
\begin{gathered}
\sum_{i} \widetilde{V}_{\alpha i} \widetilde{V}_{\beta i}^{*}=\delta_{\alpha \beta} \\
\sum_{i} \widetilde{\Delta}_{i 1} \widetilde{V}_{\alpha i} \widetilde{V}_{\beta i}^{*}=\sum_{i} \Delta_{i 1} V_{\alpha i} V_{\beta i}^{*}+\mathcal{A}_{\alpha \beta}-B \delta_{\alpha \beta} \\
\sum_{i} \widetilde{\Delta}_{i 1}\left(\widetilde{\Delta}_{i 1}+2 B\right) \widetilde{V}_{\alpha i} \widetilde{V}_{\beta i}^{*}=\sum_{i} \Delta_{i 1}\left(\Delta_{i 1}+\mathcal{A}_{\alpha \alpha}+\mathcal{A}_{\beta \beta}\right) V_{\alpha i} V_{\beta i}^{*}+\mathcal{A}_{\alpha \beta}^{2}-B^{2} \delta_{\alpha \beta} \\
\sum_{i} \widetilde{\Delta}_{i 1}\left(\widetilde{\Delta}_{i 1}^{2}+3 B \widetilde{\Delta}_{i 1}+3 B^{2}\right) \widetilde{V}_{\alpha i} \widetilde{V}_{\beta i}^{*}=\sum_{i}\left[\Delta_{i 1}^{3}+\frac{3}{2} \Delta_{i 1}^{2}\left(\mathcal{A}_{\alpha \alpha}+\mathcal{A}_{\beta \beta}\right)+\Delta_{i 1}\left(\mathcal{A}_{\alpha \alpha}^{2}\right.\right. \\
\left.\left.+\mathcal{A}_{\beta \beta}^{2}+\mathcal{A}_{\alpha \alpha} \mathcal{A}_{\beta \beta}\right)+\mathcal{A}_{\alpha \beta}^{3}\right] V_{\alpha i} V_{\beta i}^{*} \\
-B^{3} \delta_{\alpha \beta}+C_{\alpha \beta}
\end{gathered}
$$


where $\mathcal{A}_{\alpha \beta}$ denotes the $(\alpha, \beta)$ element of the matter potential matrix $\mathcal{A}=\operatorname{Diag}\left\{A, 0, A \epsilon, A^{\prime}\right\}$ and

$$
C_{\alpha \beta}=-\frac{1}{2} \sum_{m, n, \gamma} \Delta_{m n}^{2} V_{\alpha m} V_{\gamma m}^{*} V_{\gamma n} V_{\beta n}^{*} \mathcal{A}_{\gamma \gamma}
$$

with $m, n=1,2,3,4$ and $\alpha, \beta, \gamma=e, \mu, \tau, s$. According to eq. (3.8), one can directly derive the exact expressions of $\left|\widetilde{V}_{\alpha i}\right|^{2}$ and $\widetilde{V}_{\alpha i} \widetilde{V}_{\beta i}^{*}$, which have been given in refs. [38, 39]. We rewrite them as

$$
\left|\widetilde{V}_{\alpha i}\right|^{2}=\frac{1}{\prod_{k \neq i} \widetilde{\Delta}_{i k}} \sum_{j}\left(F_{\alpha}^{i j}\left|V_{\alpha j}\right|^{2}+C_{\alpha \alpha}\right)
$$

with

$$
F_{\alpha}^{i j}=\prod_{k \neq i}\left(\Delta_{j 1}-\widetilde{\Delta}_{k 1}-B+\mathcal{A}_{\alpha \alpha}\right)
$$

and

$$
\widetilde{V}_{\alpha i} \widetilde{V}_{\beta i}^{*}=\frac{1}{\prod_{k \neq i} \widetilde{\Delta}_{i k}} \sum_{j}\left(F_{\alpha \beta}^{i j} V_{\alpha j} V_{\beta j}^{*}+C_{\alpha \beta}\right)
$$

with

$$
\begin{aligned}
F_{\alpha \beta}^{i j}=\frac{1}{2} & {\left[-\left(\Delta_{j 1}-\widetilde{\Delta}_{i 1}-B\right)\left(\mathcal{A}_{\alpha \alpha}^{2}+\mathcal{A}_{\beta \beta}^{2}+4 \mathcal{A}_{\alpha \alpha} \mathcal{A}_{\beta \beta}\right)\right.} \\
& \left.+\prod_{k \neq i}\left(\Delta_{j 1}-\widetilde{\Delta}_{k 1}-B+\mathcal{A}_{\alpha \alpha}+\mathcal{A}_{\beta \beta}\right)+\prod_{k \neq i}\left(\Delta_{j 1}-\widetilde{\Delta}_{k 1}-B\right)\right],
\end{aligned}
$$

where $\alpha, \beta=e, \mu, \tau, s ; i, j, k=1,2,3,4, B$ is taken from eq. (3.7). Comparing with the formulas of $\left|\widetilde{V}_{\alpha i}\right|^{2}$ and $\widetilde{V}_{\alpha i} \widetilde{V}_{\beta i}^{*}$ in refs. [38, 39], we get rid of the uneasy terms $m_{i}^{2}-\widetilde{m}_{j}^{2}$ and include the radiative correction effects in eqs. (3.10) and (3.12). With the help of eqs. (3.4), (3.5) and (3.12), we can directly write out the probability of $\nu_{\alpha} \rightarrow \nu_{\beta}$ (for $\alpha, \beta=e, \mu, \tau, s)$ in a medium:

$$
\begin{aligned}
\widetilde{P}_{\alpha \beta}= & \delta_{\alpha \beta}-4 \sum_{i<j} \operatorname{Re}\left(\widetilde{V}_{\alpha i} \widetilde{V}_{\beta i}^{*} \widetilde{V}_{\alpha j}^{*} \widetilde{V}_{\beta j}\right) \sin ^{2}\left(\frac{\widetilde{\Delta}_{j i} L}{4 E}\right) \\
& +2 \sum_{i<j} \operatorname{Im}\left(\widetilde{V}_{\alpha i} \widetilde{V}_{\beta i}^{*} \widetilde{V}_{\alpha j}^{*} \widetilde{V}_{\beta j}\right) \sin \left(\frac{\widetilde{\Delta}_{j i} L}{2 E}\right)
\end{aligned}
$$

with $i, j=1,2,3,4$. Note that the results in eq. (1.4) and eqs. (3.1)-(3.14) only apply to a neutrino beam propagating in matter. When considering an antineutrino beam, we need to do the replacements $A \rightarrow-A, A^{\prime} \rightarrow-A^{\prime}$ and $V \rightarrow V^{*}$. Similar to the standard three-flavor mixing scheme, we discuss the $(3+1)$ flavor mixing in dense matter by considering the following four cases separately: case $\left(\mathrm{NMO}, \nu_{3+1}\right)$, case (IMO, $\left.\nu_{3+1}\right)$, case $\left(\mathrm{NMO}, \bar{\nu}_{3+1}\right)$ and case (IMO, $\bar{\nu}_{3+1}$ ). In the following discussion, $N_{e}=N_{n}=N_{p}$ (i.e., $A^{\prime}=-A / 2$ and $\epsilon \simeq 5 \times 10^{-5}$ ) is assumed and $V$ is parametrized as

$$
V=R_{34}\left(\theta_{34}, \delta_{34}\right) \cdot R_{24}\left(\theta_{24}, \delta_{24}\right) \cdot R_{14}\left(\theta_{14}, \delta_{14}\right) \cdot U,
$$

where $R_{i j}\left(\theta_{i j}, \delta_{i j}\right.$ ) (for $\left.i j=13,24,34\right)$ represent the $4 \times 4$ two-dimension rotation matrices in the $(i, j)$ complex plane with the mixing angle $\theta_{i j}$ and the CP-violating phase $\delta_{i j}$, and $U$ has been defined in eq. (2.12). Numerically, we typically take the best-fit values 

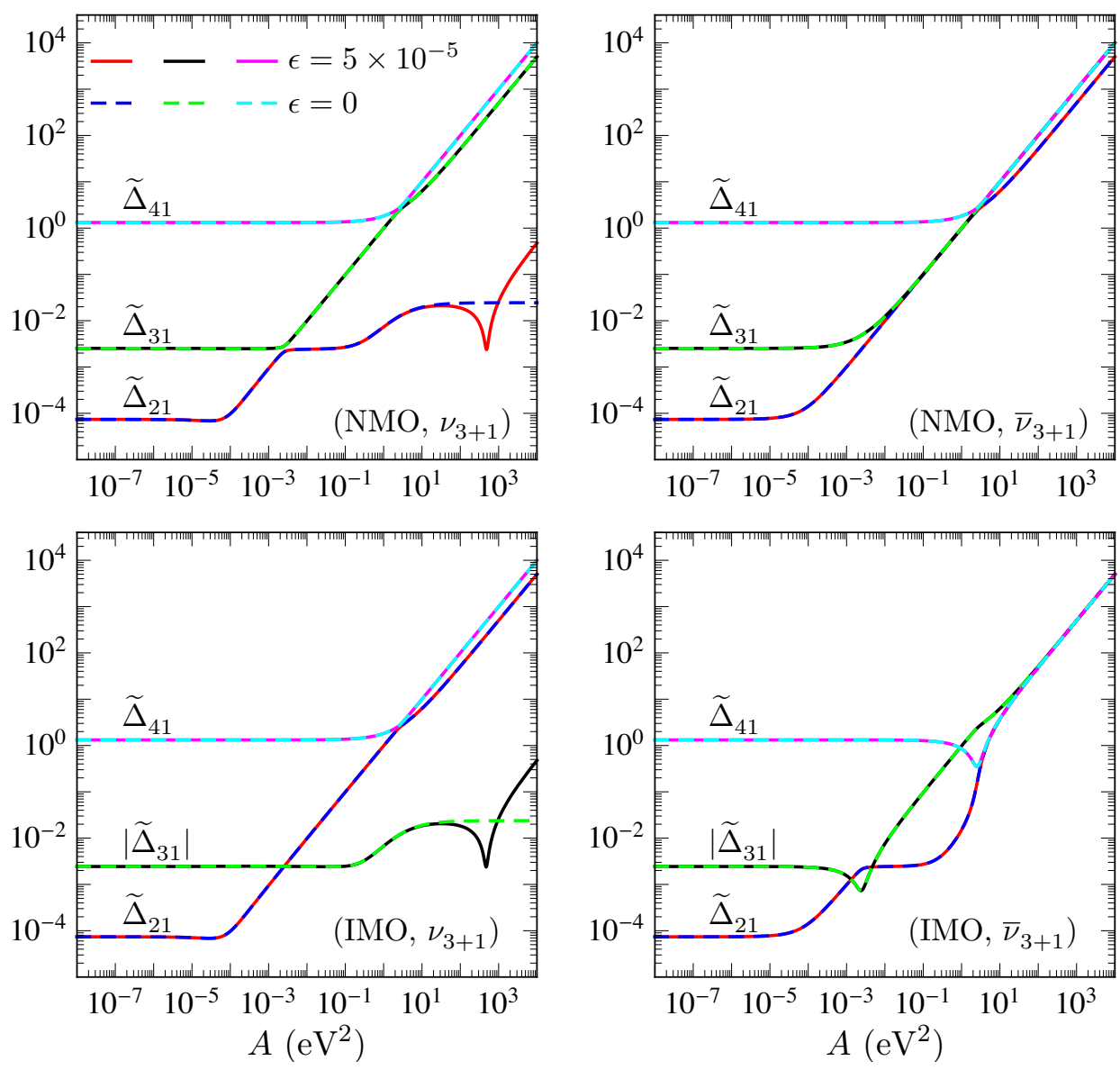

Figure 7. In the $(3+1)$ mixing scheme, the illustration of how the effective neutrino mass-squared differences $\widetilde{\Delta}_{21},\left|\widetilde{\Delta}_{31}\right|$ and $\widetilde{\Delta}_{41}$ evolve with the matter effect parameter $A$ in the case with or without radiative corrections, where we typically take the best-fit values of $\left(\theta_{12}, \theta_{13}, \theta_{23}, \delta, \Delta_{21}, \Delta_{31}\right)[36,37]$, and for the active-sterile neutrino mixing part $\left(\theta_{14}, \theta_{24}, \theta_{34}\right)=\left(6.66^{\circ}, 7.81^{\circ}, 0\right), \delta_{14}=\delta_{24}=\delta_{34}=0$, $\Delta_{41}=1.32 \mathrm{eV}^{2}[40]$.

of $\left(\theta_{12}, \theta_{13}, \theta_{23}, \delta, \Delta_{21}, \Delta_{31}\right)$ shown below eq. (2.12), and $\left(\theta_{14}, \theta_{24}, \theta_{34}\right)=\left(6.66^{\circ}, 7.81^{\circ}, 0^{\circ}\right)$, $\delta_{14}=\delta_{24}=\delta_{34}=0$ and $\Delta_{41}=1.32 \mathrm{eV}^{2}$ [40] for the active-sterile mixing part. Analytically, we expand the exact expressions of $\widetilde{\Delta}_{i j}$ (for $i j=21,31,41$ ) and $\left|\widetilde{V}_{\alpha i}\right|^{2}$ (for $\alpha=e, \mu, \tau, s$ and $i=1,2,3,4)$ in eqs. (3.4), (3.5) and (3.10) in terms of $\Delta_{21} / A, \Delta_{31} / A, \Delta_{41} / A$ and $\epsilon$. Thus the analytical approximations in this section are only valid for the $A \gg \Delta_{41}$ range.

\section{$3.1\left(\mathrm{NMO}, \nu_{3+1}\right)$}

Considering a neutrino beam with inverted mass ordering in the $(3+1)$ flavor mixing scheme, we illustrate how $\widetilde{\Delta}_{i j}$ (for $i j=21,31,41$ ) and $\left|\widetilde{V}_{\alpha i}\right|^{2}$ (for $\alpha=e, \mu, \tau, s$ and $i=1,2,3,4)$ evolve with the matter effect parameter $A$ in the upper left panel of figure 7 and figure 8 , respectively. Note that we always have $\widetilde{\Delta}_{41}>\widetilde{\Delta}_{31}>\widetilde{\Delta}_{21}$ in cases (NMO, $\left.\nu_{3+1}\right)$ and $\left(\mathrm{NMO}, \bar{\nu}_{3+1}\right)$, implying that there is no intersection in the upper row of figure 7 . Comparing figure 2 and figure 7 , the evolutions of $\left|\widetilde{V}_{\alpha i}\right|^{2}$ (for $\alpha=e, \mu, \tau$ and $i=1,2,3$ ) with $A$ in the $(3+1)$ flavor mixing scheme are similar to those of $\left|\widetilde{U}_{\alpha i}\right|^{2}$ (for $\alpha=e, \mu, \tau$ and 


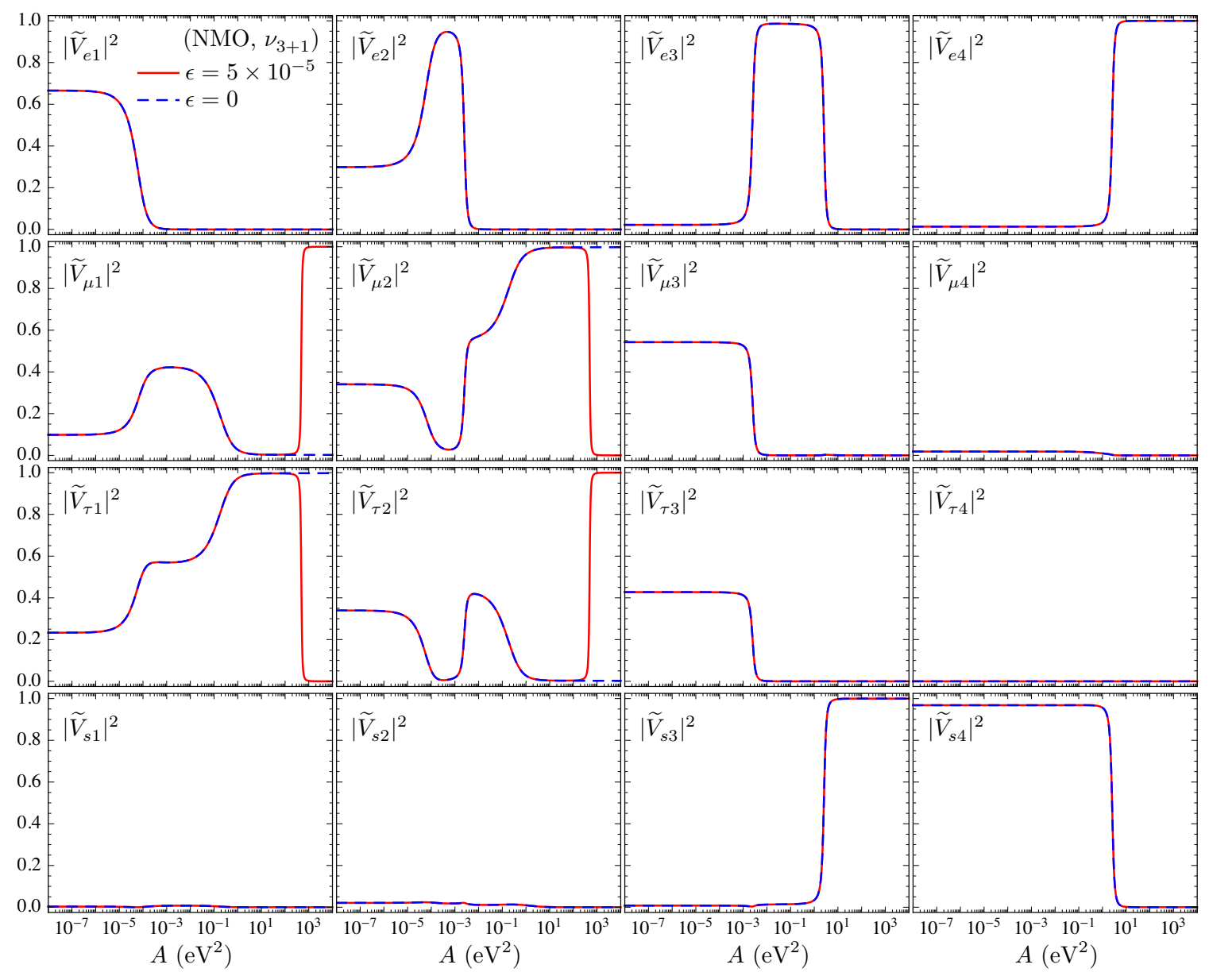

Figure 8. In the $(3+1)$ mixing scheme, the illustration of how $\left|\widetilde{V}_{\alpha i}\right|^{2}$ (for $\alpha=e, \mu, \tau, s$ and $i=$ $1,2,3,4)$ evolve with the matter effect parameter $A$ in the case $\left(\mathrm{NMO}, \nu_{3+1}\right)$ with or without radiative corrections, where we typically take the best-fit values of $\left(\theta_{12}, \theta_{13}, \theta_{23}, \delta, \Delta_{21}, \Delta_{31}\right)[36,37]$, and for the active-sterile neutrino mixing part $\left(\theta_{14}, \theta_{24}, \theta_{34}\right)=\left(6.66^{\circ}, 7.81^{\circ}, 0\right), \delta_{14}=\delta_{24}=\delta_{34}=0$, $\Delta_{41}=1.32 \mathrm{eV}^{2}[40]$.

$i=1,2,3$ ) in the standard three-flavor mixing scheme if $A$ is not big enough (for example, $A<10^{-2} \mathrm{eV}^{2}$ ). And it is the same case for $\widetilde{\Delta}_{i j}$ (for $i j=21,31$ ). However, if the matter density is very big, the neutrino flavor mixing in $(3+1)$ flavor mixing scheme can be very different from that in the standard three-flavor mixing scheme discussed in the last section. By making perturbative expansions of eq. (3.4) in terms of $\Delta_{21} / A, \Delta_{31} / A, \Delta_{41} / A$ and $\epsilon$, we get

$$
\begin{aligned}
& \widetilde{\Delta}_{21} \simeq \xi_{s}, \\
& \begin{aligned}
\widetilde{\Delta}_{31} \simeq \frac{A}{2}-\frac{1}{2}[ & A \epsilon-\xi_{s}+\left(1-\left|V_{e 2}\right|^{2}-3\left|V_{s 2}\right|^{2}\right) \Delta_{21}+\left(1-\left|V_{e 3}\right|^{2}-3\left|V_{s 3}\right|^{2}\right) \Delta_{31} \\
& \left.+\left(1-\left|V_{e 4}\right|^{2}-3\left|V_{s 4}\right|^{2}\right) \Delta_{41}\right],
\end{aligned} \\
& \begin{aligned}
\widetilde{\Delta}_{41} \simeq A-\frac{1}{2}[ & A \epsilon-\xi_{s}+\left(1-3\left|V_{e 2}\right|^{2}-\left|V_{s 2}\right|^{2}\right) \Delta_{21}+\left(1-3\left|V_{e 3}\right|^{2}-\left|V_{s 3}\right|^{2}\right) \Delta_{31} \\
& \left.+\left(1-3\left|V_{e 4}\right|^{2}-\left|V_{s 4}\right|^{2}\right) \Delta_{41}\right],
\end{aligned}
\end{aligned}
$$


where

$$
\begin{aligned}
\xi_{s}=\{ & {\left[\left(\left|V_{\mu 2}\right|^{2}+\left|V_{\tau 2}\right|^{2}\right) \Delta_{21}+\left(\left|V_{\mu 3}\right|^{2}+\left|V_{\tau 3}\right|^{2}\right) \Delta_{31}+\left(\left|V_{\mu 4}\right|^{2}+\left|V_{\tau 4}\right|^{2}\right) \Delta_{41}+A \epsilon\right]^{2} } \\
& -4 A \epsilon\left(\left|V_{\mu 2}\right|^{2} \Delta_{21}+\left|V_{\mu 3}\right|^{2} \Delta_{31}+\left|V_{\mu 4}\right|^{2} \Delta_{41}\right) \\
& \left.-4\left(\Delta_{21} \Delta_{31} C_{e s}^{14}+\Delta_{21} \Delta_{41} C_{e s}^{13}+\Delta_{31} \Delta_{41} C_{e s}^{12}\right)\right\}^{1 / 2}
\end{aligned}
$$

From eqs. (3.16) and (3.17), one can see that there are significant radiative corrections to $\widetilde{\Delta}_{21}$ for the appearance of $A \epsilon$ in the leading order. Thus $\widetilde{\Delta}_{21}$ will approximately approach $A \epsilon$ instead of a constant value if $A$ is big enough. We also notice that $\widetilde{\Delta}_{21}$ has a minimum value by observing the quadratic function of $A$ inside the brace of eq. (3.17). The corresponding expression of $A$ is

$$
A \simeq \frac{1}{\epsilon}\left[\left(\left|V_{\mu 2}\right|^{2}-\left|V_{\tau 2}\right|^{2}\right) \Delta_{21}+\left(\left|V_{\mu 3}\right|^{2}-\left|V_{\tau 3}\right|^{2}\right) \Delta_{31}+\left(\left|V_{\mu 4}\right|^{2}-\left|V_{\tau 4}\right|^{2}\right) \Delta_{41}\right]
$$

which can be simplified as $A \simeq\left(\left|V_{\mu 4}\right|^{2}-\left|V_{\tau 4}\right|^{2}\right) \Delta_{41} / \epsilon \simeq 481 \mathrm{eV}^{2}$ by considering $\Delta_{41} \gg \Delta_{31} \gg \Delta_{21}$ and the numerical input in figure 7 .

By expanding eq. (3.10) in terms of $\Delta_{21} / A, \Delta_{31} / A, \Delta_{41} / A$ and $\epsilon$, we approximately express $\left|\widetilde{V}_{\alpha i}\right|^{2}$ as

$$
\begin{aligned}
& \left|\widetilde{V}_{e 1}\right|^{2} \simeq\left|\widetilde{V}_{e 2}\right|^{2} \simeq\left|\widetilde{V}_{e 3}\right|^{2} \simeq 0, \quad\left|\widetilde{V}_{s 1}\right|^{2} \simeq\left|\widetilde{V}_{s 2}\right|^{2} \simeq\left|\widetilde{V}_{s 4}\right|^{2} \simeq 0, \quad\left|\widetilde{V}_{e 4}\right|^{2} \simeq\left|\widetilde{V}_{s 3}\right|^{2} \simeq 1 \\
& \begin{aligned}
\left|\widetilde{V}_{\mu 1}\right|^{2} \simeq\left|\widetilde{V}_{\tau 2}\right|^{2} \simeq \frac{1}{2}+\frac{1}{2 \xi_{s}}[ & {\left[A \epsilon+\Delta_{21}\left(\left|V_{\tau 2}\right|^{2}-\left|V_{\mu 2}\right|^{2}\right)+\Delta_{31}\left(\left|V_{\tau 3}\right|^{2}-\left|V_{\mu 3}\right|^{2}\right)\right.} \\
& \left.+\Delta_{41}\left(\left|V_{\tau 4}\right|^{2}-\left|V_{\mu 4}\right|^{2}\right)\right]
\end{aligned} \\
& \begin{aligned}
\left|\widetilde{V}_{\mu 2}\right|^{2} \simeq\left|\widetilde{V}_{\tau 1}\right|^{2} \simeq \frac{1}{2}-\frac{1}{2 \xi_{s}} & {\left[A \epsilon+\Delta_{21}\left(\left|V_{\tau 2}\right|^{2}-\left|V_{\mu 2}\right|^{2}\right)+\Delta_{31}\left(\left|V_{\tau 3}\right|^{2}-\left|V_{\mu 3}\right|^{2}\right)\right.} \\
& \left.+\Delta_{41}\left(\left|V_{\tau 4}\right|^{2}-\left|V_{\mu 4}\right|^{2}\right)\right]
\end{aligned} \\
& \left|\widetilde{V}_{\mu 3}\right|^{2} \simeq\left|\widetilde{V}_{\mu 4}\right|^{2} \simeq\left|\widetilde{V}_{\tau 3}\right|^{2} \simeq\left|\widetilde{V}_{\tau 4}\right|^{2} \simeq 0 .
\end{aligned}
$$

Namely, the neutrino flavor mixing matrix $\widetilde{V}$ can be approximately described by one degree of freedom,

$$
\left.\widetilde{V}\right|_{A \gg \Delta_{41}} \simeq\left(\begin{array}{cccc}
0 & 0 & 0 & 1 \\
\cos \theta_{s} & \sin \theta_{s} & 0 & 0 \\
-\sin \theta_{s} & \cos \theta_{s} & 0 & 0 \\
0 & 0 & 1 & 0
\end{array}\right),
$$

where $\theta_{s} \in[0, \pi / 2]$ and $\tan ^{2} \theta_{s} \simeq\left|\widetilde{V}_{\mu 2}\right|^{2} /\left|\widetilde{V}_{\mu 1}\right|^{2}$ with $\left|\widetilde{V}_{\mu 1}\right|^{2}$ and $\left|\widetilde{V}_{\mu 2}\right|^{2}$ being taken from eq. (3.19). The corresponding neutrino oscillation probabilities $\widetilde{P}_{\alpha \beta}$ (for $\left.\alpha \beta=e, \mu, \tau, s\right)$ in eq. (3.14) are reduced to

$$
\begin{aligned}
& \widetilde{P}_{e e} \simeq 1, \quad \widetilde{P}_{e \mu} \simeq 0, \quad \widetilde{P}_{e \tau} \simeq 0, \quad \widetilde{P}_{e s} \simeq 0, \\
& \widetilde{P}_{\mu e} \simeq 0, \quad \widetilde{P}_{\mu \mu} \simeq 1-\sin ^{2} 2 \theta_{s} \sin ^{2} \frac{\widetilde{\Delta}_{21} L}{4 E}, \quad \widetilde{P}_{\mu \tau} \simeq \sin ^{2} 2 \theta_{s} \sin ^{2} \frac{\widetilde{\Delta}_{21} L}{4 E}, \quad \widetilde{P}_{\mu s} \simeq 0, \\
& \widetilde{P}_{\tau e} \simeq 0, \quad \widetilde{P}_{\tau \mu} \simeq \sin ^{2} 2 \theta_{s} \sin ^{2} \frac{\widetilde{\Delta}_{21} L}{4 E}, \quad \widetilde{P}_{\tau \tau} \simeq 1-\sin ^{2} 2 \theta_{s} \sin ^{2} \frac{\widetilde{\Delta}_{21} L}{4 E}, \quad \widetilde{P}_{\tau s} \simeq 0, \\
& \widetilde{P}_{s e} \simeq 0, \quad \widetilde{P}_{s \mu} \simeq 0, \quad \widetilde{P}_{s \tau} \simeq 0, \quad \widetilde{P}_{s s} \simeq 1,
\end{aligned}
$$




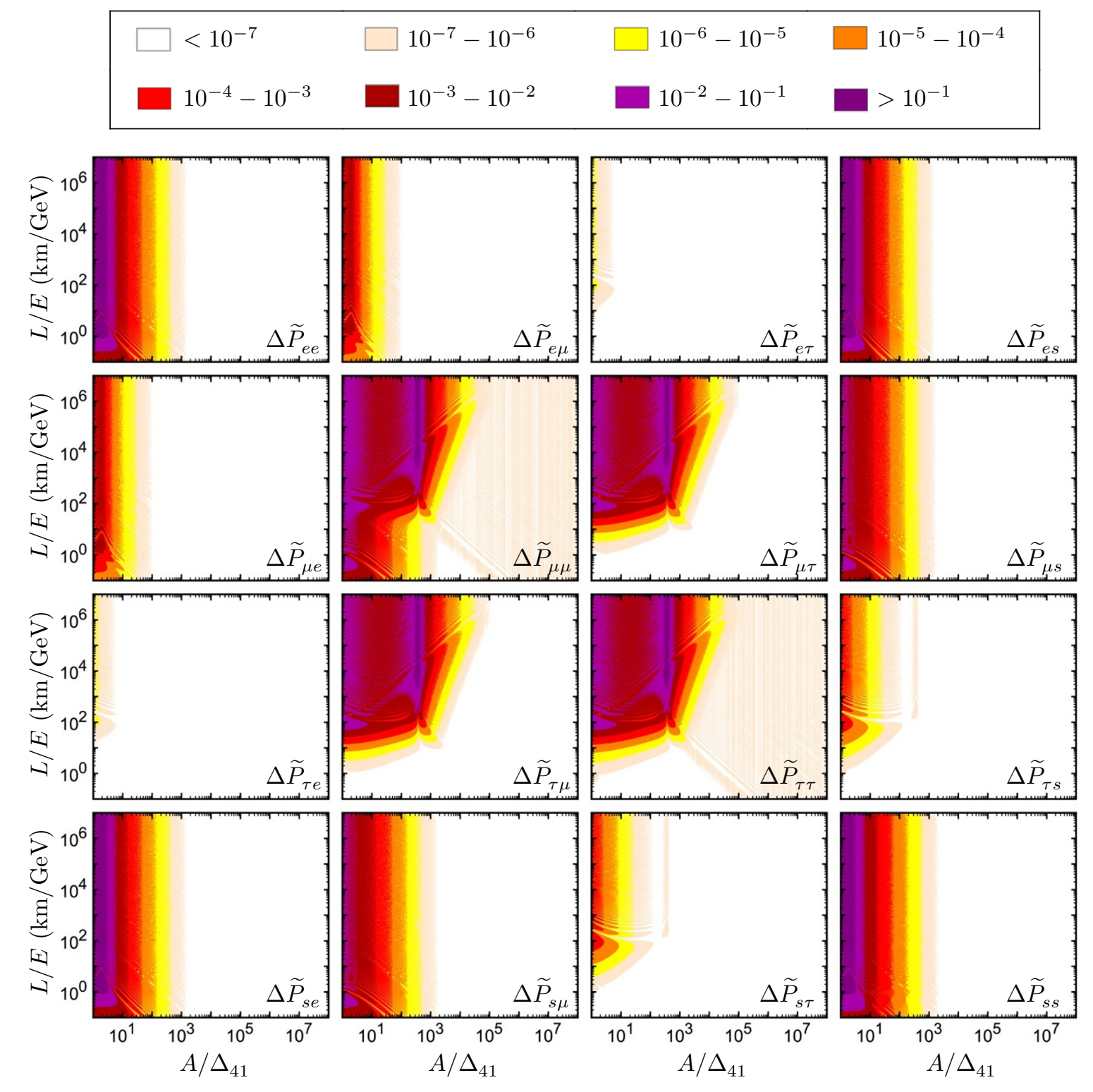

Figure 9. The absolute errors of our analytical approximations in eq. (3.21), where the best-fit values of $\left(\theta_{12}, \theta_{13}, \theta_{23}, \delta, \Delta_{21}, \Delta_{31}\right)$ in refs. [36, 37] have been input, $\left(\theta_{14}, \theta_{24}, \theta_{34}\right)=\left(6.66^{\circ}, 7.81^{\circ}, 0\right)$, $\delta_{14}=\delta_{24}=\delta_{34}=0, \Delta_{41}=1.32 \mathrm{eV}^{2}[40]$ and $\epsilon \simeq 5 \times 10^{-5}[26]$.

where $\widetilde{\Delta}_{21}$ has been given in eq. (3.16) and $\sin ^{2} 2 \theta_{s}=\left|\widetilde{V}_{\mu 1}\right|^{2}\left(1-\left|\widetilde{V}_{\mu 1}\right|^{2}\right)$ with $\left|\widetilde{V}_{\mu 1}\right|^{2}$ being derived in eq. (3.19). The absolute errors of $\widetilde{P}_{\alpha \beta}$ are illustrated in figure 9 , from which we can see that eq. (3.21) is a good approximation in a wide range of $L / E$ and $A / \Delta_{41}$. For the upper left part in each subgraph of figure 9 , the accuracies are not good enough and the largest errors in $\Delta \widetilde{P}_{\mu \mu}, \Delta \widetilde{P}_{\mu \tau}, \Delta \widetilde{P}_{\tau \mu}$ and $\Delta \widetilde{P}_{\tau \tau}$ appear around the minimum of $\widetilde{\Delta}_{21}\left(A / \Delta_{41} \sim 4 \times 10^{2}\right)$. This can be improved by keeping higher orders of $\Delta_{21} / A, \Delta_{31} / A$, $\Delta_{41} / A$ and $\epsilon$ or just making perturbative expansions in terms of $\Delta_{21} / A, \Delta_{31} / A$ and $\epsilon$. 


\begin{tabular}{|c|c|c|c|c|}
\hline & $\left(\mathrm{NMO}, \nu_{3+1}\right)$ & $\left(\mathrm{IMO}, \nu_{3+1}\right)$ & $\left(\mathrm{NMO}, \bar{\nu}_{3+1}\right)$ & $\left(\mathrm{IMO}, \bar{\nu}_{3+1}\right)$ \\
\hline$\widetilde{\Delta}_{21}$ & $A \epsilon$ & $A / 2-A \epsilon$ & $A / 2$ & $A / 2-A \epsilon$ \\
\hline$\widetilde{\Delta}_{31}$ & $A / 2$ & $-A \epsilon$ & $A-A \epsilon$ & $-A / 2$ \\
\hline$\widetilde{\Delta}_{41}$ & $A$ & $A-A \epsilon$ & $A$ & $A / 2$ \\
\hline$\widetilde{V}$ & $\left(\begin{array}{llll}0 & 0 & 0 & 1 \\
1 & 0 & 0 & 0 \\
0 & 1 & 0 & 0 \\
0 & 0 & 1 & 0\end{array}\right)$ & $\left(\begin{array}{llll}0 & 0 & 0 & 1 \\
0 & 0 & 1 & 0 \\
1 & 0 & 0 & 0 \\
0 & 1 & 0 & 0\end{array}\right)$ & $\left(\begin{array}{llll}1 & 0 & 0 & 0 \\
0 & 0 & 0 & 1 \\
0 & 0 & 1 & 0 \\
0 & 1 & 0 & 0\end{array}\right)$ & $\left(\begin{array}{llll}0 & 0 & 1 & 0 \\
0 & 0 & 0 & 1 \\
0 & 1 & 0 & 0 \\
1 & 0 & 0 & 0\end{array}\right)$ \\
\hline
\end{tabular}

Table 2. In the $(3+1)$ mixing scheme, the analytical expressions of $\widetilde{\Delta}_{j i}($ for $j i=21,31,41)$ and $\widetilde{V}$ in the $A \rightarrow \infty$ limit, where we only show the terms of order $\mathcal{O}(A)$ for $\widetilde{\Delta}_{j i}$.

Specifically, if $A \epsilon$ is negligible in eqs. (3.16) and (3.19), one may abandon the smaller terms of $\Delta_{21}$ and $\Delta_{31}$, and get

$$
\begin{aligned}
\widetilde{\Delta}_{21} & \simeq\left(\left|V_{\mu 4}\right|^{2}+\left|V_{\tau 4}\right|^{2}\right) \Delta_{41}, \\
\left|\widetilde{V}_{\mu 1}\right|^{2} & \simeq\left|\widetilde{V}_{\tau 2}\right|^{2} \simeq \frac{\left|V_{\tau 4}\right|^{2}}{\left|V_{\mu 4}\right|^{2}+\left|V_{\tau 4}\right|^{2}}, \\
\left|\widetilde{V}_{\mu 2}\right|^{2} & \simeq\left|\widetilde{V}_{\tau 1}\right|^{2} \simeq \frac{\left|V_{\mu 4}\right|^{2}}{\left|V_{\mu 4}\right|^{2}+\left|V_{\tau 4}\right|^{2}},
\end{aligned}
$$

where $\left|V_{\mu 4}\right|^{2}=\cos ^{2} \theta_{14} \sin ^{2} \theta_{24},\left|V_{\tau 4}\right|^{2}=\cos ^{2} \theta_{14} \cos ^{2} \theta_{24} \sin ^{2} \theta_{34}$ from the parametrization of $V$ in eq. (3.15). This is equivalent to the asymptotic behaviors of $\widetilde{\Delta}_{21}$ and $\left|\widetilde{V}_{\alpha i}\right|^{2}$ (for $\alpha i=\mu 1, \mu 2, \tau 1, \tau 2)$ in very dense matter in the case without radiative corrections (i.e. the blue dashed lines in figure 8). Due to the typical value $\theta_{34}=0$ inputted in figure 8 , we get $\left|\widetilde{V}_{\mu 1}\right|^{2} \simeq\left|\widetilde{V}_{\tau 2}\right|^{2} \simeq 0$ and $\left|\widetilde{V}_{\mu 2}\right|^{2} \simeq\left|\widetilde{V}_{\tau 1}\right|^{2} \simeq 1$. By choosing a non-zero value of $\theta_{34}$, the neutrino flavor mixing can be very different. With the increase of $A$, the $A \epsilon$ term in eqs. (3.16) and (3.19) will become dominate. In the $A \rightarrow \infty$ limit, $\left|\widetilde{V}_{\mu 1}\right|^{2} \simeq\left|\widetilde{V}_{\tau 2}\right|^{2} \simeq 1$ and $\left|\widetilde{V}_{\mu 2}\right|^{2} \simeq\left|\widetilde{V}_{\tau 1}\right|^{2} \simeq 0$ can be derived from eq. (3.19). Considering this extreme case, we summarize the corresponding analytical expressions of $\widetilde{\Delta}_{j i}$ (for $\left.j i=21,31,41\right)$ and $\widetilde{V}$ in table 2, where the four cases $\left(\mathrm{NMO}, \nu_{3+1}\right),\left(\mathrm{IMO}, \nu_{3+1}\right),\left(\mathrm{NMO}, \bar{\nu}_{3+1}\right)$ and (IMO, $\left.\bar{\nu}_{3+1}\right)$ are all considered separately. With $\left|\widetilde{V}_{\alpha i}\right|^{2}$ taking 0 or 1 (or $\theta_{s} \rightarrow 0$ or $\pi / 2$ ), there will be no neutrino oscillation between the four flavors and it makes no sense to discuss lepton flavor mixing.

\section{$3.2\left(\mathrm{IMO}, \nu_{3+1}\right)$}

Given a neutrino beam with inverted mass ordering in the $(3+1)$ flavor mixing scheme, the evolutions of $\widetilde{\Delta}_{i j}$ (for $i j=21,31,41$ ) and $\left|\widetilde{V}_{\alpha i}\right|^{2}$ (for $\alpha=e, \mu, \tau, s$ and $i=1,2,3,4$ ) with $A$ are demonstrated in the lower left panel of figure 7 and figure 10, respectively. In this case, $\widetilde{\Delta}_{31}<0<\widetilde{\Delta}_{21}<\widetilde{\Delta}_{41}$ always holds from figure 7 . By making perturbative expansions of 


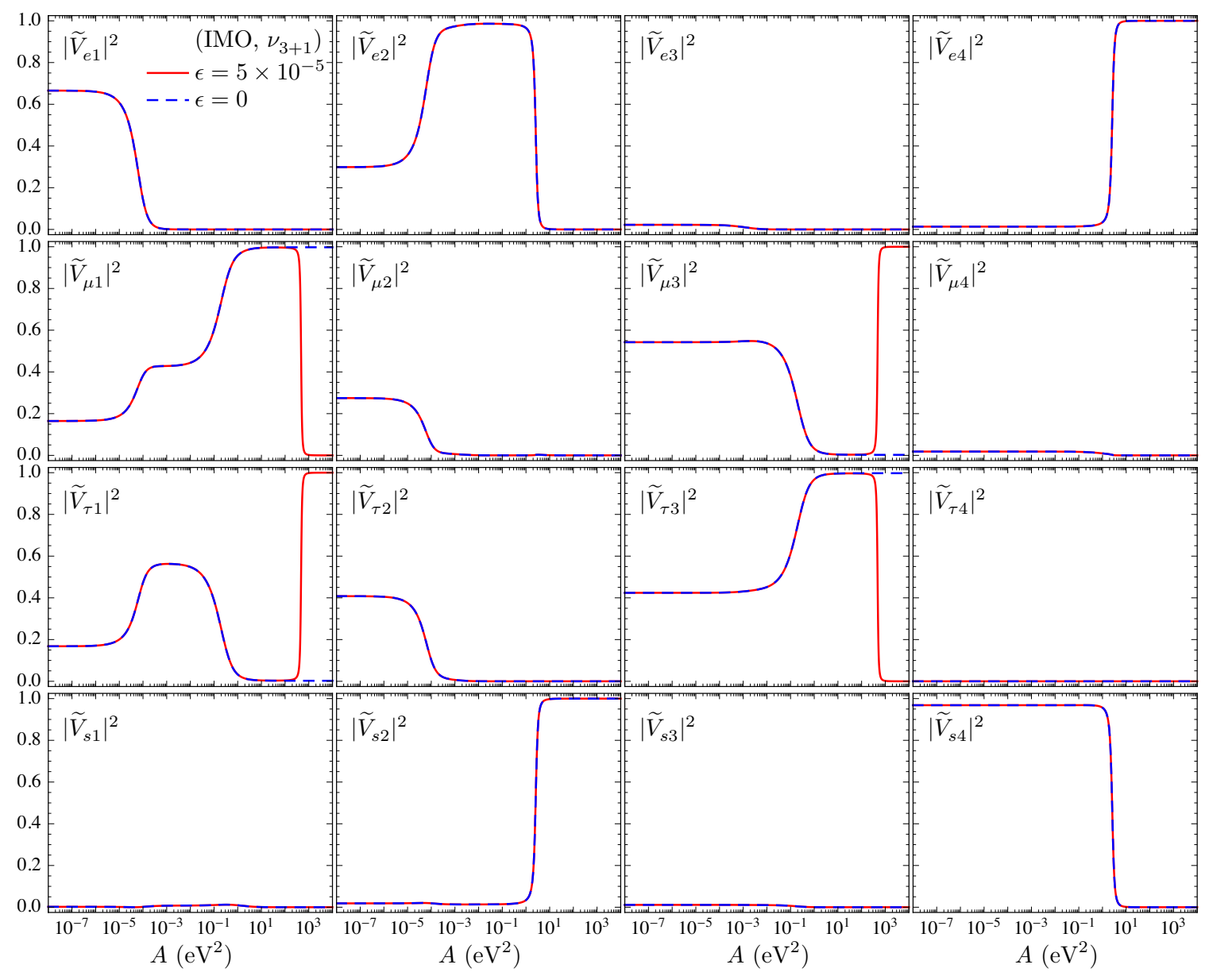

Figure 10. In the $(3+1)$ mixing scheme, the illustration of how $\left|\widetilde{V}_{\alpha i}\right|^{2}$ (for $\alpha=e, \mu, \tau, s$ and $i=$ $1,2,3,4)$ evolve with the matter effect parameter $A$ in the case (IMO, $\nu_{3+1}$ ) with or without radiative corrections, where we typically take the best-fit values of $\left(\theta_{12}, \theta_{13}, \theta_{23}, \delta, \Delta_{21}, \Delta_{31}\right)[36,37]$, and for the active-sterile neutrino mixing part $\left(\theta_{14}, \theta_{24}, \theta_{34}\right)=\left(6.66^{\circ}, 7.81^{\circ}, 0\right), \delta_{14}=\delta_{24}=\delta_{34}=0$, $\Delta_{41}=1.32 \mathrm{eV}^{2}[40]$.

eq. (3.5), we get

$$
\begin{aligned}
& \begin{aligned}
\widetilde{\Delta}_{21} \simeq \frac{A}{2}-\frac{1}{2}[ & A \epsilon+\xi_{s}+\left(1-\left|V_{e 2}\right|^{2}-3\left|V_{s 2}\right|^{2}\right) \Delta_{21}+\left(1-\left|V_{e 3}\right|^{2}-3\left|V_{s 3}\right|^{2}\right) \Delta_{31} \\
& \left.+\left(1-\left|V_{e 4}\right|^{2}-3\left|V_{s 4}\right|^{2}\right) \Delta_{41}\right], \\
\widetilde{\Delta}_{31} \simeq-\xi_{s}, & \\
\widetilde{\Delta}_{41} \simeq A-\frac{1}{2}[ & A \epsilon+\xi_{s}+\left(1-3\left|V_{e 2}\right|^{2}-\left|V_{s 2}\right|^{2}\right) \Delta_{21}+\left(1-3\left|V_{e 3}\right|^{2}-\left|V_{s 3}\right|^{2}\right) \Delta_{31} \\
& \left.+\left(1-3\left|V_{e 4}\right|^{2}-\left|V_{s 4}\right|^{2}\right) \Delta_{41}\right]
\end{aligned}
\end{aligned}
$$

where $\xi_{s}$ has been defined in eq. (3.17). From eq. (3.23), it is easy to see that there are significant radiative corrections to $\widetilde{\Delta}_{31}$ with the $\epsilon$ term appearing in the leading order. There are a minimum of $\widetilde{\Delta}_{31}$ corresponding to $A$ in eq. (3.18). Similarly, eq. (3.10) can be 
reduced to

$$
\begin{aligned}
& \left|\widetilde{V}_{e 1}\right|^{2} \simeq\left|\widetilde{V}_{e 2}\right|^{2} \simeq\left|\widetilde{V}_{e 3}\right|^{2} \simeq 0, \quad\left|\widetilde{V}_{s 1}\right|^{2} \simeq\left|\widetilde{V}_{s 3}\right|^{2} \simeq\left|\widetilde{V}_{s 4}\right|^{2} \simeq 0, \quad\left|\widetilde{V}_{e 4}\right|^{2} \simeq\left|\widetilde{V}_{s 2}\right|^{2} \simeq 1 \\
& \begin{aligned}
\left|\widetilde{V}_{\mu 1}\right|^{2} \simeq\left|\widetilde{V}_{\tau 3}\right|^{2} \simeq \frac{1}{2}-\frac{1}{2 \xi_{s}} & {\left[A \epsilon+\Delta_{21}\left(\left|V_{\tau 2}\right|^{2}-\left|V_{\mu 2}\right|^{2}\right)+\Delta_{31}\left(\left|V_{\tau 3}\right|^{2}-\left|V_{\mu 3}\right|^{2}\right)\right.} \\
& \left.+\Delta_{41}\left(\left|V_{\tau 4}\right|^{2}-\left|V_{\mu 4}\right|^{2}\right)\right]
\end{aligned} \\
& \begin{aligned}
\left|\widetilde{V}_{\mu 3}\right|^{2} \simeq\left|\widetilde{V}_{\tau 1}\right|^{2} \simeq \frac{1}{2}+\frac{1}{2 \xi_{s}}[ & A \epsilon+\Delta_{21}\left(\left|V_{\tau 2}\right|^{2}-\left|V_{\mu 2}\right|^{2}\right)+\Delta_{31}\left(\left|V_{\tau 3}\right|^{2}-\left|V_{\mu 3}\right|^{2}\right) \\
& \left.+\Delta_{41}\left(\left|V_{\tau 4}\right|^{2}-\left|V_{\mu 4}\right|^{2}\right)\right]
\end{aligned} \\
& \left|\widetilde{V}_{\mu 2}\right|^{2} \simeq\left|\widetilde{V}_{\mu 4}\right|^{2} \simeq\left|\widetilde{V}_{\tau 2}\right|^{2} \simeq\left|\widetilde{V}_{\tau 4}\right|^{2} \simeq 0 .
\end{aligned}
$$

This means $\widetilde{V}$ can be approximately parametrized as

$$
\left.\widetilde{V}\right|_{A \gg \Delta_{41}} \simeq\left(\begin{array}{cccc}
0 & 0 & 0 & 1 \\
\cos \theta_{s} & 0 & \sin \theta_{s} & 0 \\
-\sin \theta_{s} & 0 & \cos \theta_{s} & 0 \\
0 & 1 & 0 & 0
\end{array}\right)
$$

where $\theta_{s} \in[0, \pi / 2]$ and $\tan ^{2} \theta_{s}=\left|\widetilde{V}_{\mu 3}\right|^{2} /\left|\widetilde{V}_{\mu 1}\right|^{2}$ with $\left|\widetilde{V}_{\mu 1}\right|^{2}$ and $\left|\widetilde{V}_{\mu 3}\right|^{2}$ having been shown in eq. (3.24). One can derive the corresponding approximate neutrino oscillation probability by substituting $\widetilde{\Delta}_{31}$ for $\widetilde{\Delta}_{21}$ in eq. (3.21). Similar to the standard three-flavor mixing scheme, it is impossible to discriminate between the normal mass ordering and the inverted mass ordering from neutrino oscillations in the $(3+1)$ flavor mixing scheme if the matter density is very big.

Note that if the $A \epsilon$ term in eqs. (3.23) and (3.24) is negligible, we omit the smaller terms of $\Delta_{21}$ and $\Delta_{31}$ and obtain

$$
\begin{aligned}
\widetilde{\Delta}_{31} & \simeq-\left(\left|V_{\mu 4}\right|^{2}+\left|V_{\tau 4}\right|^{2}\right) \Delta_{41}, \\
\left|\widetilde{V}_{\mu 1}\right|^{2} & \simeq\left|\widetilde{V}_{\tau 3}\right|^{2} \simeq \frac{\left|V_{\mu 4}\right|^{2}}{\left|V_{\mu 4}\right|^{2}+\left|V_{\tau 4}\right|^{2}}, \\
\left|\widetilde{V}_{\mu 3}\right|^{2} & \simeq\left|\widetilde{V}_{\tau 1}\right|^{2} \simeq \frac{\left|V_{\tau 4}\right|^{2}}{\left|V_{\mu 4}\right|^{2}+\left|V_{\tau 4}\right|^{2}},
\end{aligned}
$$

which is consistent with the asymptotic behaviors of $\widetilde{\Delta}_{31}$ and $\left|\widetilde{V}_{\alpha i}\right|^{2}$ (for $\alpha i=\mu 1, \mu 3, \tau 1, \tau 3$ ) in very dense matter in the case without radiative corrections (i.e. the blue dashed line in figure 10). By inputting $\theta_{34}=0$ (i.e., $\left|V_{\tau 4}\right|^{2}=0$ ), we directly get $\left|\widetilde{V}_{\mu 1}\right|^{2} \simeq\left|\widetilde{V}_{\tau 3}\right|^{2} \simeq 1$ and $\left|\widetilde{V}_{\mu 3}\right|^{2} \simeq\left|\widetilde{V}_{\tau 1}\right|^{2} \simeq 0$ from eq. (3.26). If the limit of $A \rightarrow \infty$ is taken, one can infer from eq. (3.24) that $\left|\widetilde{V}_{\mu 1}\right|^{2} \simeq\left|\widetilde{V}_{\tau 3}\right|^{2} \simeq 0$ and $\left|\widetilde{V}_{\mu 3}\right|^{2} \simeq\left|\widetilde{V}_{\tau 1}\right|^{2} \simeq 1$, leading to no neutrino oscillations.

\section{$3.3\left(\mathrm{NMO}, \bar{\nu}_{3+1}\right)$}

When it comes to a neutrino beam with normal mass ordering in the $(3+1)$ flavor mixing scheme, the corresponding evolutions of $\widetilde{\Delta}_{i j}$ (for $\left.i j=21,31,41\right)$ and $\left|\widetilde{V}_{\alpha i}\right|^{2}$ (for $\alpha=e, \mu, \tau, s$ 


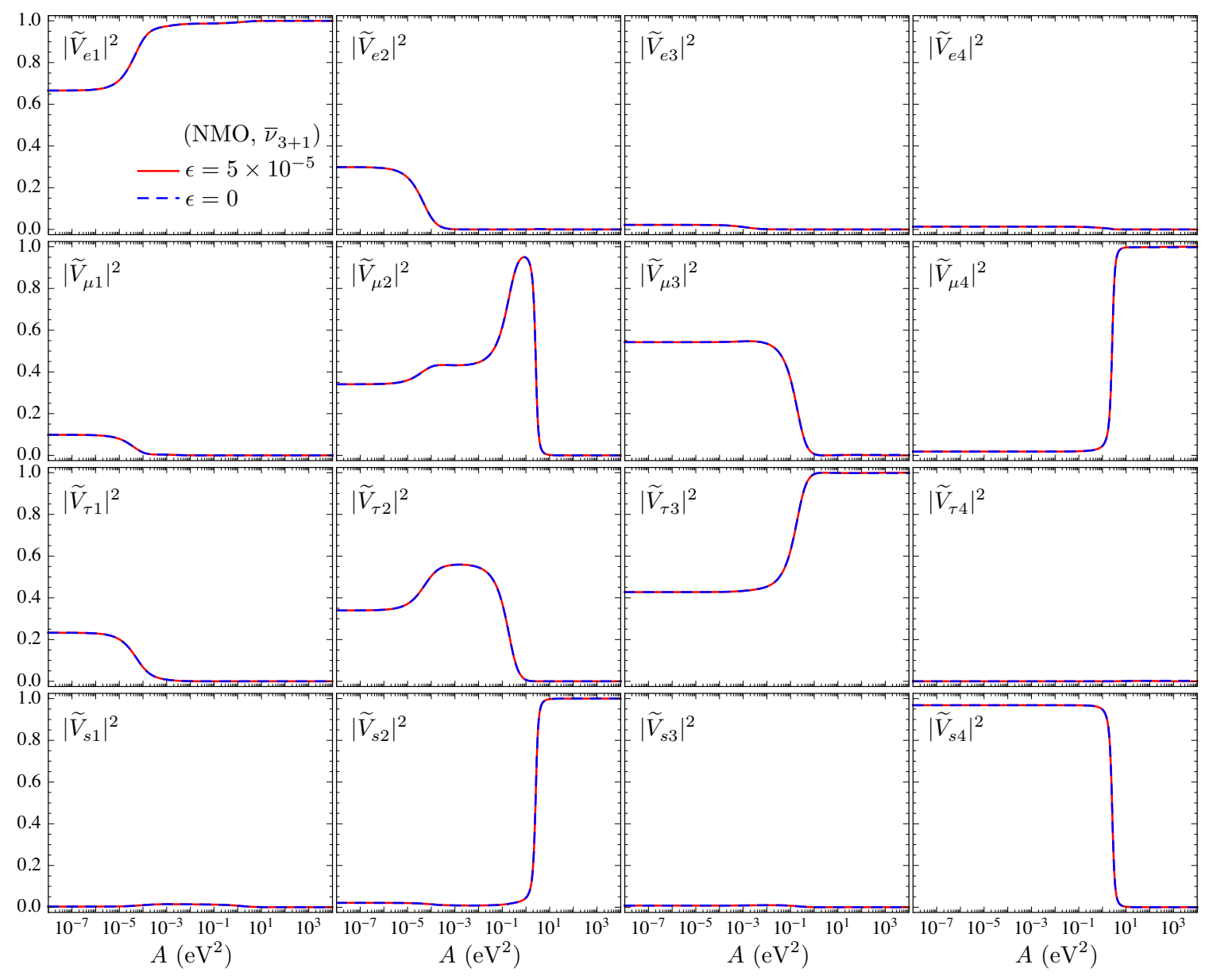

Figure 11. In the $(3+1)$ mixing scheme, the illustration of how $\left|\widetilde{V}_{\alpha i}\right|^{2}$ (for $\alpha=e, \mu, \tau, s$ and $i=$ $1,2,3,4)$ evolve with the matter effect parameter $A$ in the case $\left(\mathrm{NMO}, \bar{\nu}_{3+1}\right)$ with or without radiative corrections, where we typically take the best-fit values of $\left(\theta_{12}, \theta_{13}, \theta_{23}, \delta, \Delta_{21}, \Delta_{31}\right)[36,37]$, and for the active-sterile neutrino mixing part $\left(\theta_{14}, \theta_{24}, \theta_{34}\right)=\left(6.66^{\circ}, 7.81^{\circ}, 0\right), \delta_{14}=\delta_{24}=\delta_{34}=0$, $\Delta_{41}=1.32 \mathrm{eV}^{2}[40]$.

and $i=1,2,3,4)$ with the matter effect parameter $A$ are illustrated in the upper right panel of figure 7 and figure 11, respectively. Analytically, we perform perturbative expansions of $\widetilde{\Delta}_{i j}$ (for $i j=21,31,41$ ) in eq. (3.4) and get

$$
\begin{aligned}
& \widetilde{\Delta}_{21} \simeq \frac{A}{2}-\frac{1}{2}\left[\left(\left|V_{e 2}\right|^{2}-\left|V_{s 2}\right|^{2}\right) \Delta_{21}+\left(\left|V_{e 3}\right|^{2}-\left|V_{s 3}\right|^{2}\right) \Delta_{31}+\left(\left|V_{e 4}\right|^{2}-\left|V_{s 4}\right|^{2}\right) \Delta_{41}\right], \\
& \widetilde{\Delta}_{31} \simeq A-\frac{1}{2}\left[A \epsilon+\xi_{s}-\left(1-3\left|V_{e 2}\right|^{2}-\left|V_{s 2}\right|^{2}\right) \Delta_{21}-\left(1-3\left|V_{e 3}\right|^{2}-\left|V_{s 3}\right|^{2}\right) \Delta_{31}\right. \\
& \left.-\left(1-3\left|V_{e 4}\right|^{2}-\left|V_{s 4}\right|^{2}\right) \Delta_{41}\right] \text {, } \\
& \widetilde{\Delta}_{41} \simeq A-\frac{1}{2}\left[A \epsilon-\xi_{s}-\left(1-3\left|V_{e 2}\right|^{2}-\left|V_{s 2}\right|^{2}\right) \Delta_{21}-\left(1-3\left|V_{e 3}\right|^{2}-\left|V_{s 3}\right|^{2}\right) \Delta_{31}\right. \\
& \left.-\left(1-3\left|V_{e 4}\right|^{2}-\left|V_{s 4}\right|^{2}\right) \Delta_{41}\right] \text {, }
\end{aligned}
$$


where

$$
\begin{aligned}
\xi_{s}= & \left\{\left[\left(\left|V_{\mu 2}\right|^{2}+\left|V_{\tau 2}\right|^{2}\right) \Delta_{21}+\left(\left|V_{\mu 3}\right|^{2}+\left|V_{\tau 3}\right|^{2}\right) \Delta_{31}+\left(\left|V_{\mu 4}\right|^{2}+\left|V_{\tau 4}\right|^{2}\right) \Delta_{41}-A \epsilon\right]^{2}\right. \\
& +4 A \epsilon\left(\left|V_{\mu 2}\right|^{2} \Delta_{21}+\left|V_{\mu 3}\right|^{2} \Delta_{31}+\left|V_{\mu 4}\right|^{2} \Delta_{41}\right) \\
& \left.-4\left(\Delta_{21} \Delta_{31} C_{e s}^{14}+\Delta_{21} \Delta_{41} C_{e s}^{13}+\Delta_{31} \Delta_{41} C_{e s}^{12}\right)\right\}^{1 / 2} .
\end{aligned}
$$

Note that there are no significant radiative corrections to $\widetilde{\Delta}_{21}, \widetilde{\Delta}_{31}$ and $\widetilde{\Delta}_{41}$ with the $A \epsilon$ term appearing in the next-to-leading order or higher order. Similarly, $\left|\widetilde{V}_{\alpha i}\right|^{2}$ can be reduced to

$$
\begin{aligned}
& \left|\widetilde{V}_{e 2}\right|^{2} \simeq\left|\widetilde{V}_{e 3}\right|^{2} \simeq\left|\widetilde{V}_{e 4}\right|^{2} \simeq 0, \quad\left|\widetilde{V}_{s 1}\right|^{2} \simeq\left|\widetilde{V}_{s 3}\right|^{2} \simeq\left|\widetilde{V}_{s 4}\right|^{2} \simeq 0, \quad\left|\widetilde{V}_{e 1}\right|^{2} \simeq\left|\widetilde{V}_{s 2}\right|^{2} \simeq 1 \\
& \begin{aligned}
\left|\widetilde{V}_{\mu 3}\right|^{2} \simeq\left|\widetilde{V}_{\tau 4}\right|^{2} \simeq \frac{1}{2}+\frac{1}{2 \xi_{s}}[ & {\left[-A \epsilon+\Delta_{21}\left(\left|V_{\tau 2}\right|^{2}-\left|V_{\mu 2}\right|^{2}\right)+\Delta_{31}\left(\left|V_{\tau 3}\right|^{2}-\left|V_{\mu 3}\right|^{2}\right)\right.} \\
& \left.+\Delta_{41}\left(\left|V_{\tau 4}\right|^{2}-\left|V_{\mu 4}\right|^{2}\right)\right]
\end{aligned} \\
& \begin{array}{c}
\left|\widetilde{V}_{\mu 4}\right|^{2} \simeq\left|\widetilde{V}_{\tau 3}\right|^{2} \simeq \frac{1}{2}-\frac{1}{2 \xi_{s}}\left[-A \epsilon+\Delta_{21}\left(\left|V_{\tau 2}\right|^{2}-\left|V_{\mu 2}\right|^{2}\right)+\Delta_{31}\left(\left|V_{\tau 3}\right|^{2}-\left|V_{\mu 3}\right|^{2}\right)\right. \\
\left.\quad+\Delta_{41}\left(\left|V_{\tau 4}\right|^{2}-\left|V_{\mu 4}\right|^{2}\right)\right]
\end{array} \\
& \left|\widetilde{V}_{\mu 1}\right|^{2} \simeq\left|\widetilde{V}_{\mu 2}\right|^{2} \simeq\left|\widetilde{V}_{\tau 1}\right|^{2} \simeq\left|\widetilde{V}_{\tau 2}\right|^{2} \simeq 0 .
\end{aligned}
$$

Namely,

$$
\left.\widetilde{V}\right|_{A \gg \Delta_{41}} \simeq\left(\begin{array}{cccc}
1 & 0 & 0 & 0 \\
0 & 0 & \cos \theta_{s} & \sin \theta_{s} \\
0 & 0 & -\sin \theta_{s} & \cos \theta_{s} \\
0 & 1 & 0 & 0
\end{array}\right),
$$

where $\theta_{s} \in[0, \pi / 2]$ and $\tan ^{2} \theta_{s}=\left|\widetilde{V}_{\mu 4}\right|^{2} /\left|\widetilde{V}_{\mu 3}\right|^{2}$ with $\left|\widetilde{V}_{\mu 3}\right|^{2}$ and $\left|\widetilde{V}_{\mu 4}\right|^{2}$ being expressed in eq. (3.29). The corresponding approximate formulas of neutrino oscillation probabilities can be obtained by doing the replacement $\widetilde{\Delta}_{21} \rightarrow \widetilde{\Delta}_{43}$ in eq. (3.21). And we have $\widetilde{\Delta}_{43} \simeq \xi_{s}$ from eq. (3.27). If the $A \epsilon$ term is not dominant in eq. (3.27), one can ignore the smaller terms of $A \epsilon, \Delta_{21}$ and $\Delta_{31}$ and get

$$
\begin{aligned}
& \left|\widetilde{V}_{\mu 3}\right|^{2} \simeq\left|\widetilde{V}_{\tau 4}\right|^{2} \simeq \frac{\left|V_{\tau 4}\right|^{2}}{\left|V_{\mu 4}\right|^{2}+\left|V_{\tau 4}\right|^{2}}, \\
& \left|\widetilde{V}_{\mu 4}\right|^{2} \simeq\left|\widetilde{V}_{\tau 3}\right|^{2} \simeq \frac{\left|V_{\mu 4}\right|^{2}}{\left|V_{\mu 4}\right|^{2}+\left|V_{\tau 4}\right|^{2}},
\end{aligned}
$$

which are equivalent to the fixed values of $\left|\widetilde{V}_{\alpha i}\right|^{2}$ (for $\alpha i=\mu 3, \mu 4, \tau 3, \tau 4$ ) in the $A \rightarrow \infty$ limit when the radiative corrections are not included. By inputting $\theta_{34}=0$ (i.e., $\left|V_{\tau 4}\right|^{2}=$ 0 ), eq. (3.31) turns into $\left|\widetilde{V}_{\mu 3}\right|^{2} \simeq\left|\widetilde{V}_{\tau 4}\right|^{2} \simeq 0$ and $\left|\widetilde{V}_{\mu 4}\right|^{2} \simeq\left|\widetilde{V}_{\tau 3}\right|^{2} \simeq 1$. In the $A \rightarrow \infty$ limit, we also have the same results: $\left|\widetilde{V}_{\mu 3}\right|^{2} \simeq\left|\widetilde{V}_{\tau 4}\right|^{2} \simeq 0$ and $\left|\widetilde{V}_{\mu 4}\right|^{2} \simeq\left|\widetilde{V}_{\tau 3}\right|^{2} \simeq 1$ with $\xi_{s} \rightarrow A \epsilon$ in eq. (3.29). Thus there will be no neutrino oscillations in the $A \rightarrow \infty$ limit with $\theta_{s} \simeq \arctan \left(\left|\widetilde{V}_{\mu 4}\right| /\left|\widetilde{V}_{\mu 3}\right|\right) \simeq \pi / 2$. Note that there is no distinguishable difference between the cases with or without radiative corrections in figure 11. However, by inputting a nonzero value of $\theta_{34}$, one may see significant radiative corrections to neutrino flavor mixing matrix elements, especially to $\widetilde{V}_{\alpha i}$ (for $\alpha i=\mu 3, \mu 4, \tau 3, \tau 4$ ) when $A$ is big enough. 


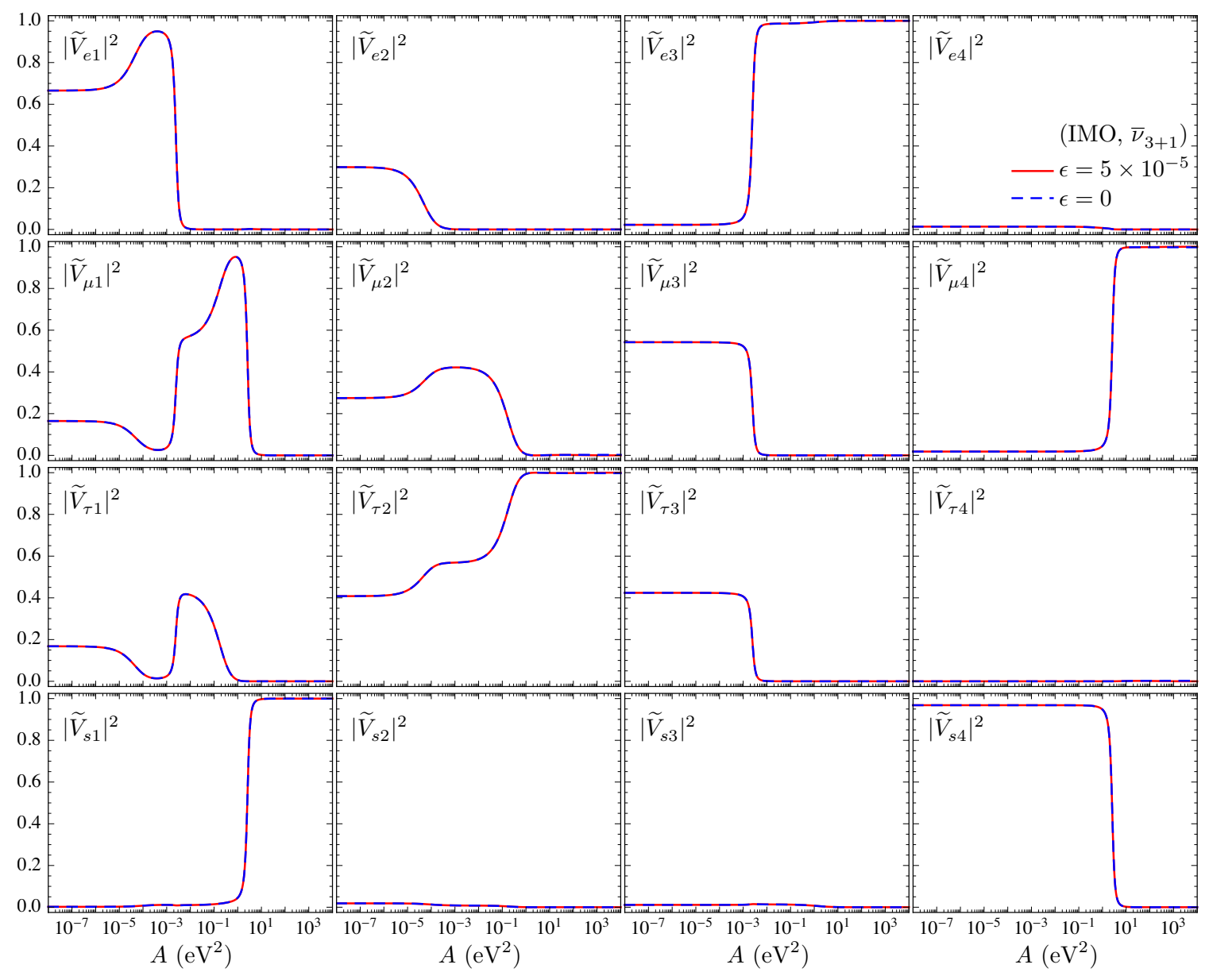

Figure 12. In the $(3+1)$ mixing scheme, the illustration of how $\left|\widetilde{V}_{\alpha i}\right|^{2}$ (for $\alpha=e, \mu, \tau, s$ and $i=$ $1,2,3,4)$ evolve with the matter effect parameter $A$ in the case (IMO, $\left.\bar{\nu}_{3+1}\right)$ with or without radiative corrections, where we typically take the best-fit values of $\left(\theta_{12}, \theta_{13}, \theta_{23}, \delta, \Delta_{21}, \Delta_{31}\right)[36,37]$, and for the active-sterile neutrino mixing part $\left(\theta_{14}, \theta_{24}, \theta_{34}\right)=\left(6.66^{\circ}, 7.81^{\circ}, 0\right), \delta_{14}=\delta_{24}=\delta_{34}=0$, $\Delta_{41}=1.32 \mathrm{eV}^{2}[40]$.

\section{$3.4\left(\mathrm{IMO}, \bar{\nu}_{3+1}\right)$}

Similarly, let us discuss the case of an antineutrino beam with inverted mass ordering in the $(3+1)$ flavor mixing scheme. The evolutions of $\widetilde{\Delta}_{i j}$ (for $\left.i j=21,31,41\right)$ and $\left|\widetilde{V}_{\alpha i}\right|^{2}$ (for $\alpha=e, \mu, \tau, s$ and $i=1,2,3,4)$ with the matter effect parameter $A$ in this case are illustrated in the lower right panel of figure 7 and figure 12, respectively. Expanding eq. (3.5) in terms of $\Delta_{21} / A, \Delta_{31} / A, \Delta_{41} / A$ and $\epsilon$, we arrive at

$$
\begin{gathered}
\widetilde{\Delta}_{21} \simeq \frac{A}{2}-\frac{1}{2}\left[A \epsilon+\xi_{s}-\left(1-\left|V_{e 2}\right|^{2}-3\left|V_{s 2}\right|^{2}\right) \Delta_{21}-\left(1-\left|V_{e 3}\right|^{2}-3\left|V_{s 3}\right|^{2}\right) \Delta_{31}\right. \\
\left.-\left(1-\left|V_{e 4}\right|^{2}-3\left|V_{s 4}\right|^{2}\right) \Delta_{41}\right], \\
\widetilde{\Delta}_{31} \simeq-\frac{A}{2}+\left(\left|V_{e 2}\right|^{2}-\left|V_{s 2}\right|^{2}\right) \Delta_{21}+\left(\left|V_{e 3}\right|^{2}-\left|V_{s 3}\right|^{2}\right) \Delta_{31}+\left(\left|V_{e 4}\right|^{2}-\left|V_{s 4}\right|^{2}\right) \Delta_{41} \\
\widetilde{\Delta}_{41} \simeq \frac{A}{2}-\frac{1}{2}\left[A \epsilon-\xi_{s}-\left(1-\left|V_{e 2}\right|^{2}-3\left|V_{s 2}\right|^{2}\right) \Delta_{21}-\left(1-\left|V_{e 3}\right|^{2}-3\left|V_{s 3}\right|^{2}\right) \Delta_{31}\right. \\
\left.-\left(1-\left|V_{e 4}\right|^{2}-3\left|V_{s 4}\right|^{2}\right) \Delta_{41}\right],
\end{gathered}
$$


with $\xi_{s}$ being defined in eq. (3.28). According to figure 7 and eq. (3.32), we always have $\widetilde{\Delta}_{31}<0<\widetilde{\Delta}_{21}<\widetilde{\Delta}_{41}$ and in the $A \rightarrow \infty$ limit, $\left|\widetilde{\Delta}_{31}\right| \simeq \widetilde{\Delta}_{41}>\widetilde{\Delta}_{21}$ holds. Similarly, the analytical approximations of $\left|\widetilde{V}_{\alpha i}\right|^{2}$ read as

$$
\begin{aligned}
& \left|\widetilde{V}_{e 1}\right|^{2} \simeq\left|\widetilde{V}_{e 2}\right|^{2} \simeq\left|\widetilde{V}_{e 4}\right|^{2} \simeq 0, \quad\left|\widetilde{V}_{s 2}\right|^{2} \simeq\left|\widetilde{V}_{s 3}\right|^{2} \simeq\left|\widetilde{V}_{s 4}\right|^{2} \simeq 0, \quad\left|\widetilde{V}_{e 3}\right|^{2} \simeq\left|\widetilde{V}_{s 1}\right|^{2} \simeq 1 \\
& \begin{aligned}
\left|\widetilde{V}_{\mu 2}\right|^{2} \simeq\left|\widetilde{V}_{\tau 4}\right|^{2} \simeq \frac{1}{2}+\frac{1}{2 \xi_{s}}[ & -A \epsilon+\Delta_{21}\left(\left|V_{\tau 2}\right|^{2}-\left|V_{\mu 2}\right|^{2}\right)+\Delta_{31}\left(\left|V_{\tau 3}\right|^{2}-\left|V_{\mu 3}\right|^{2}\right) \\
& \left.+\Delta_{41}\left(\left|V_{\tau 4}\right|^{2}-\left|V_{\mu 4}\right|^{2}\right)\right]
\end{aligned} \\
& \begin{aligned}
\left|\widetilde{V}_{\mu 4}\right|^{2} \simeq\left|\widetilde{V}_{\tau 2}\right|^{2} \simeq \frac{1}{2}-\frac{1}{2 \xi_{s}} & {\left[-A \epsilon+\Delta_{21}\left(\left|V_{\tau 2}\right|^{2}-\left|V_{\mu 2}\right|^{2}\right)+\Delta_{31}\left(\left|V_{\tau 3}\right|^{2}-\left|V_{\mu 3}\right|^{2}\right)\right.} \\
& \left.+\Delta_{41}\left(\left|V_{\tau 4}\right|^{2}-\left|V_{\mu 4}\right|^{2}\right)\right]
\end{aligned} \\
& \left|\widetilde{V}_{\mu 1}\right|^{2} \simeq\left|\widetilde{V}_{\mu 3}\right|^{2} \simeq\left|\widetilde{V}_{\tau 1}\right|^{2} \simeq\left|\widetilde{V}_{\tau 3}\right|^{2} \simeq 0
\end{aligned}
$$

which implies

$$
\left.\widetilde{V}\right|_{A \gg \Delta_{41}} \simeq\left(\begin{array}{cccc}
0 & 0 & 1 & 0 \\
0 & \cos \theta_{s} & 0 & \sin \theta_{s} \\
0 & -\sin \theta_{s} & 0 & \cos \theta_{s} \\
1 & 0 & 0 & 0
\end{array}\right)
$$

where $\theta_{s} \in[0, \pi / 2]$ and $\tan ^{2} \theta_{s}=\left|\widetilde{V}_{\mu 4}\right|^{2} /\left|\widetilde{V}_{\mu 2}\right|^{2}$ with $\left|\widetilde{V}_{\mu 2}\right|^{2}$ and $\left|\widetilde{V}_{\mu 4}\right|^{2}$ being expressed in eq. (3.33). One can directly write out the corresponding approximations of neutrino oscillation probability by replacing $\widetilde{\Delta}_{21}$ in eq. (3.21) with $\widetilde{\Delta}_{42} \simeq \xi_{s}$ from eq. (3.32). If the smaller terms of $A \epsilon$ in eq. (3.33) is negligible, we obtain

$$
\begin{aligned}
& \left|\widetilde{V}_{\mu 2}\right|^{2} \simeq\left|\widetilde{V}_{\tau 4}\right|^{2} \simeq \frac{\left|V_{\tau 4}\right|^{2}}{\left|V_{\mu 4}\right|^{2}+\left|V_{\tau 4}\right|^{2}}, \\
& \left|\widetilde{V}_{\mu 4}\right|^{2} \simeq\left|\widetilde{V}_{\tau 2}\right|^{2} \simeq \frac{\left|V_{\mu 4}\right|^{2}}{\left|V_{\mu 4}\right|^{2}+\left|V_{\tau 4}\right|^{2}}
\end{aligned}
$$

after throwing out the smaller terms of $\Delta_{21}$ and $\Delta_{31}$. By inputting $\theta_{34}=0$ taken in figure 12, eq. (3.35) turns out to be $\left|\widetilde{V}_{\mu 2}\right|^{2} \simeq\left|\widetilde{V}_{\tau 4}\right|^{2} \simeq 0$ and $\left|\widetilde{V}_{\mu 4}\right|^{2} \simeq\left|\widetilde{V}_{\tau 2}\right|^{2} \simeq 1$. In the $A \rightarrow \infty$ limit, we get $\left|\widetilde{V}_{\mu 2}\right|^{2} \simeq\left|\widetilde{V}_{\tau 4}\right|^{2} \simeq 0$ and $\left|\widetilde{V}_{\mu 4}\right|^{2} \simeq\left|\widetilde{V}_{\tau 2}\right|^{2} \simeq 1$ no matter which value $\theta_{34}$ takes. Thus no neutrino oscillations between the four flavors will happen with $\theta_{s} \simeq$ $\arctan \left(\left|\widetilde{V}_{\mu 4}\right| /\left|\widetilde{V}_{\mu 2}\right|\right) \simeq \pi / 2$. Note that the neutrino flavor mixing with radiative corrections can be very different from the case without radiative corrections if $\theta_{34}$ is non-zero.

\section{Summary}

With the coming of the precision measurement era of neutrino physics, we are committed to digging the underlying physics behind the lepton flavor mixing [41] and on the other hand to conducting cosmological and astronomical researches with neutrinos being a good probe. As preliminarily discussed in ref. [25], it is possible to explore the density and size of a hidden compact object in the universe by observing its effects on the neutrino flavor 
mixing. In this paper, we point out that radiative corrections to the matter potentials can significantly affect the neutrino flavor mixing in dense matter. Considering the standard three-flavor mixing scheme with radiative corrections, we derive the exact expressions of the effective neutrino mass-squared differences $\widetilde{\Delta}_{i j}$, the moduli square of the nine lepton flavor mixing matrix elements $\left|\widetilde{U}_{\alpha i}\right|^{2}$, the vector sides of the Dirac leptonic unitarity triangles $\widetilde{U}_{\alpha i} \widetilde{U}_{\beta i}^{*}$ in a medium. From these exact formulas, the neutrino flavor mixing in dense matter are numerically and analytically discussed. Different from the fixed value of $\left|\widetilde{U}_{\alpha i}\right|^{2}$ in dense matter in the case without radiative corrections, $\left|\widetilde{U}_{\alpha i}\right|^{2}$ can be very sensitive to the value of $A$ and trivially approach 0 or 1 in the $A \rightarrow \infty$ limit if radiative corrections are taken into account. When it comes to the $(3+1)$ flavor mixing scheme, the neutrino flavor mixing will be very different from the standard three-flavor scheme if $\mathrm{A}$ is big enough but not infinite. However it is meaningless to discuss the lepton flavor mixing in both schemes in the $A \rightarrow \infty$ limit.

\section{Acknowledgments}

We would like to thank Prof. Zhi-zhong Xing and Prof. Shun Zhou for their inspirations on this topic. We are also indebted to Di Zhang for useful discussions. This work is supported partly by the National Natural Science Foundation of China under grant No. 11835005 and No. 11947227.

Open Access. This article is distributed under the terms of the Creative Commons Attribution License (CC-BY 4.0), which permits any use, distribution and reproduction in any medium, provided the original author(s) and source are credited.

\section{References}

[1] L. Wolfenstein, Neutrino Oscillations in Matter, Phys. Rev. D 17 (1978) 2369 [InSPIRE].

[2] S.P. Mikheev and A.Y. Smirnov, Resonant amplification of neutrino oscillations in matter and solar neutrino spectroscopy, Nuovo Cim. C 9 (1986) 17 [INSPIRE].

[3] Particle Data Group collaboration, Review of Particle Physics, Phys. Rev. D 98 (2018) 030001 [INSPIRE].

[4] M. Freund, Analytic approximations for three neutrino oscillation parameters and probabilities in matter, Phys. Rev. D 64 (2001) 053003 [hep-ph/0103300] [INSPIRE].

[5] E.K. Akhmedov, R. Johansson, M. Lindner, T. Ohlsson and T. Schwetz, Series expansions for three flavor neutrino oscillation probabilities in matter, JHEP 04 (2004) 078 [hep-ph/0402175] [INSPIRE].

[6] H.-J. He and X.-J. Xu, Connecting Leptonic Unitarity Triangle to Neutrino Oscillation, Phys. Rev. D 89 (2014) 073002 [arXiv:1311.4496] [InSPIRE].

[7] X.-J. Xu, Why is the neutrino oscillation formula expanded in $\Delta m_{21}^{2} / \Delta m_{31}^{2}$ still accurate near the solar resonance in matter?, JHEP 10 (2015) 090 [arXiv: 1502.02503] [INSPIRE].

[8] H.-J. He and X.-J. Xu, Connecting the leptonic unitarity triangle to neutrino oscillation with CP-violation in the vacuum and in matter, Phys. Rev. D 95 (2017) 033002 [arXiv: 1606. 04054] [INSPIRE]. 
[9] Z.-z. Xing and J.-y. Zhu, Analytical approximations for matter effects on CP-violation in the accelerator-based neutrino oscillations with $E \lesssim 1$ GeV, JHEP 07 (2016) 011 [arXiv: 1603.02002] [INSPIRE].

[10] P.B. Denton, H. Minakata and S.J. Parke, Compact Perturbative Expressions For Neutrino Oscillations in Matter, JHEP 06 (2016) 051 [arXiv: 1604.08167] [INSPIRE].

[11] Y.-F. Li, Y. Wang and Z.-z. Xing, Terrestrial matter effects on reactor antineutrino oscillations at JUNO or RENO-50: how small is small?, Chin. Phys. C 40 (2016) 091001 [arXiv: 1605.00900] [INSPIRE].

[12] Y.-F. Li, J. Zhang, S. Zhou and J.-y. Zhu, Looking into Analytical Approximations for Three-flavor Neutrino Oscillation Probabilities in Matter, JHEP 12 (2016) 109 [arXiv: 1610.04133] [INSPIRE].

[13] A. Ioannisian and S. Pokorski, Three Neutrino Oscillations in Matter, Phys. Lett. B 782 (2018) 641 [arXiv : 1801.10488] [INSPIRE].

[14] Y.-F. Li, Z.-z. Xing and J.-y. Zhu, Indirect unitarity violation entangled with matter effects in reactor antineutrino oscillations, Phys. Lett. B 782 (2018) 578 [arXiv:1802.04964] [INSPIRE].

[15] P.B. Denton, S.J. Parke and X. Zhang, Rotations Versus Perturbative Expansions for Calculating Neutrino Oscillation Probabilities in Matter, Phys. Rev. D 98 (2018) 033001 [arXiv: 1806.01277] [INSPIRE].

[16] S.T. Petcov and Y.-L. Zhou, On Neutrino Mixing in Matter and CP and T Violation Effects in Neutrino Oscillations, Phys. Lett. B 785 (2018) 95 [arXiv:1806.09112] [InSPIRE].

[17] X. Wang and S. Zhou, On the Properties of the Effective Jarlskog Invariant for Three-flavor Neutrino Oscillations in Matter, Nucl. Phys. B 950 (2020) 114867 [arXiv:1908.07304] [INSPIRE].

[18] S. Zhou, Symmetric formulation of neutrino oscillations in matter and its intrinsic connection to renormalization-group equations, J. Phys. G 44 (2017) 044006 [arXiv: 1612.03537] [INSPIRE].

[19] S.H. Chiu and T.K. Kuo, Features of Neutrino Mixing, Phys. Rev. D 97 (2018) 055026 [arXiv: 1712.08487] [INSPIRE].

[20] Z.-z. Xing, S. Zhou and Y.-L. Zhou, Renormalization-Group Equations of Neutrino Masses and Flavor Mixing Parameters in Matter, JHEP 05 (2018) 015 [arXiv:1802.00990] [INSPIRE].

[21] X. Wang and S. Zhou, Analytical solutions to renormalization-group equations of effective neutrino masses and mixing parameters in matter, JHEP 05 (2019) 035 [arXiv:1901.10882] [INSPIRE].

[22] M. Blennow and T. Ohlsson, Effective neutrino mixing and oscillations in dense matter, Phys. Lett. B 609 (2005) 330 [hep-ph/0409061] [INSPIRE].

[23] G.-y. Huang, J.-H. Liu and S. Zhou, Matter effects on the flavor conversions of solar neutrinos and high-energy astrophysical neutrinos, Nucl. Phys. B 931 (2018) 324 [arXiv: 1803.02037] [INSPIRE].

[24] Z.-Z. Xing and J.-Y. Zhu, Sum rules and asymptotic behaviors of neutrino mixing in dense matter, Nucl. Phys. B 949 (2019) 114803 [arXiv:1905.08644] [INSPIRE]. 
[25] S. Luo, Neutrino Oscillation in Dense Matter, Phys. Rev. D 101 (2020) 033005 [arXiv: 1911.06301] [INSPIRE].

[26] F.J. Botella, C.S. Lim and W.J. Marciano, Radiative Corrections to Neutrino Indices of Refraction, Phys. Rev. D 35 (1987) 896 [InSPIRE].

[27] S. Böser et al., Status of Light Sterile Neutrino Searches, Prog. Part. Nucl. Phys. 111 (2020) 103736 [arXiv: 1906.01739] [INSPIRE].

[28] V.D. Barger, K. Whisnant, S. Pakvasa and R.J.N. Phillips, Matter Effects on Three-Neutrino Oscillations, Phys. Rev. D 22 (1980) 2718 [inSPIRE].

[29] H.W. Zaglauer and K.H. Schwarzer, The Mixing Angles in Matter for Three Generations of Neutrinos and the MSW Mechanism, Z. Phys. C 40 (1988) 273 [INSPIRE].

[30] Z.-z. Xing, New formulation of matter effects on neutrino mixing and CP-violation, Phys. Lett. B 487 (2000) 327 [hep-ph/0002246] [INSPIRE].

[31] V.A. Naumov, Three neutrino oscillations in matter, CP-violation and topological phases, Int. J. Mod. Phys. D 1 (1992) 379 [InSPIRE].

[32] C. Jarlskog, Commutator of the Quark Mass Matrices in the Standard Electroweak Model and a Measure of Maximal CP-violation, Phys. Rev. Lett. 55 (1985) 1039 [InSPIRE].

[33] Z.-z. Xing, Flavor mixing and CP-violation of massive neutrinos, Int. J. Mod. Phys. A 19 (2004) 1 [hep-ph/0307359] [INSPIRE].

[34] H. Zhang and Z.-z. Xing, Leptonic unitarity triangles in matter, Eur. Phys. J. C 41 (2005) 143 [hep-ph/0411183] [INSPIRE].

[35] Z.-z. Xing and H. Zhang, Reconstruction of the neutrino mixing matrix and leptonic unitarity triangles from long-baseline neutrino oscillations, Phys. Lett. B 618 (2005) 131 [hep-ph/0503118] [INSPIRE].

[36] I. Esteban, M.C. Gonzalez-Garcia, A. Hernandez-Cabezudo, M. Maltoni and T. Schwetz, Global analysis of three-flavour neutrino oscillations: synergies and tensions in the determination of $\theta_{23}, \delta_{C P}$ and the mass ordering, JHEP 01 (2019) 106 [arXiv:1811.05487] [INSPIRE].

[37] NuFIT 4.1 (2019), www.nu-fit.org.

[38] W. Li, J. Ling, F. Xu and B. Yue, Matter Effect of Light Sterile Neutrino: An Exact Analytical Approach, JHEP 10 (2018) 021 [arXiv: 1808. 03985] [INSPIRE].

[39] H. Zhang, Sum rules of four-neutrino mixing in matter, Mod. Phys. Lett. A 22 (2007) 1341 [hep-ph/0606040] [INSPIRE].

[40] A. Diaz, C.A. Argüelles, G.H. Collin, J.M. Conrad and M.H. Shaevitz, Where Are We With Light Sterile Neutrinos?, arXiv:1906.00045 [INSPIRE].

[41] Z.-z. Xing, Flavor structures of charged fermions and massive neutrinos, Phys. Rept. 854 (2020) 1 [arXiv: 1909.09610] [INSPIRE]. 